\title{
Electricity derivatives pricing with forward-looking information is
}

\author{
Roland Füss $^{\mathrm{a}, \mathrm{b}}$, Steffen Mahringer ${ }^{\mathrm{c}}$, Marcel Prokopczuk ${ }^{\mathrm{d}, \mathrm{e}, *}$ \\ a Swiss Institute of Banking and Finance (s/bf), University of St. Gallen, Rosenbergstr. 52, 9000 St. Gallen, Switzerland \\ ${ }^{\mathrm{b}}$ Centre for European Research (ZEW), Mannheim, Germany \\ ${ }^{\mathrm{c}}$ University of St. Gallen, Rosenbergstr. 52, 9000 St. Gallen, Switzerland \\ d School of Economics and Management, Leibniz University Hannover, Königsworther Platz 1, 30167 Hannover, Germany \\ e ICMA Centre, Henley Business School, University of Reading, RG6 6BA, UK
}

\section{A R T I C L E I N F O}

\section{Article history:}

Received 24 February 2014

Received in revised form

15 April 2015

Accepted 28 May 2015

Available online 11 June 2015

JEL classification:

G12

G13

Q4

Q41

Keywords:

Electricity futures

Fundamental model

Derivatives pricing

Forward-looking information

Enlargement of filtrations

\begin{abstract}
A B S T R A C T
In order to increase overall transparency on key operational information, power transmission system operators publish an increasing amount of fundamental data, including forecasts of electricity demand and available capacity. We employ a fundamental model for electricity prices which lends itself well to integrating such forecasts, while retaining ease of implementation and tractability to allow for analytic derivatives pricing formulae. In an extensive futures pricing study, the pricing performance of our model is shown to further improve based on the inclusion of electricity demand and capacity forecasts, thus confirming the general importance of forward-looking information for electricity derivatives pricing. However, we also find that the usefulness of integrating forecast data into the pricing approach is primarily limited to those periods during which electricity prices are highly sensitive to demand or available capacity, whereas the impact is less visible when fuel prices are the primary underlying driver to prices instead.
\end{abstract}

(c) 2015 Elsevier B.V. All rights reserved.

\section{Introduction}

Following the liberalization of electricity markets in many countries, utility companies and other market participants have been facing an increasing need for new pricing models in order to accurately and efficiently evaluate spot and derivative electricity contracts. In addition, the end of cost-based pricing and the transition towards deregulated markets also gave rise to new financial risks, threatening to impose substantial losses especially for sellers of electricity forward contracts. As such, the necessity to now optimize against the market for both standard electricity products and tailored contingent claims additionally required effective and integrated risk management strategies to be developed.

\footnotetext{
We have benefited from the helpful comments of Fred Espen Benth, Michael Coulon, Enrico De Giorgi, Karl Frauendorfer, and participants at the Conference on Energy Finance 2012, Trondheim, the Conference of the Swiss Society for Financial Market Research 2013, Zurich, the Eastern Finance Association Meeting 2013, St. Pete Beach, the Financial Management Association Conference 2013, Luxembourg, and at the ICMA Centre 2013 Research Seminar, Reading. We also thank Richard Frape from Marex Spectron and Richard Price from National Grid for providing data as well as explanations on the intricacies of the British electricity market.

* Corresponding author at: School of Economics and Management, Leibniz University Hannover, Königsworther Platz 1, 30167 Hannover, Germanny.

E-mail addresses: roland.fuess@unisg.ch (R. Füss), steffen.mahringer@t-online.de (S. Mahringer), prokopczuk@fmt.uni-hannover.de (M. Prokopczuk).
} 
These developments have to be seen in the context of the unique behavior of electricity (spot) prices, which is primarily induced by the non-storability of this commodity: apart from hydropower with limited storage capabilities, an exact matching of flow supply and flow demand for electricity is required at every point in time. The resulting price dynamics with their well-known stylized facts such as spikiness, mean-reversion, and seasonality, have extensively been analyzed in the literature, ${ }^{1}$ yet still pose a challenge to both practitioners and researchers in terms of adequately modeling their trajectories.

However, the non-storability of electricity has further implications for the price formation mechanism. First, unlike in a classic storage economy, it is the instantaneous nature of electricity that causes the intertemporal linkages between economic agents' decisions today and tomorrow to break down. In fact, this forms the basis for electricity markets usually being characterized as very transparent with respect to their underlying economic factors, including electricity demand, available levels of generation capacity, as well as the costs for generating fuels and emissions allowances. Against this background, structural approaches taking this information explicitly into account appear especially appealing to electricity price modeling (see, e.g., Pirrong, 2012). Second, and as the above implies, the classic assumption that the evolution of all relevant pricing information, i.e., the information filtration, is fully determined by the price process of the commodity itself, does not hold for non-storable assets such as electricity. In other words, today's electricity prices do not necessarily reflect forward-looking information that is publicly available to all market participants. ${ }^{2}$ At the same time, legal requirements and voluntary initiatives to increase data transparency have had power transmission system operators (TSOs) publish an increasing amount of data regarding the condition of their network, including, e.g., forecasts about expected electricity demand or updated schedules of planned short-term outages. ${ }^{3}$ Pricing electricity spot and derivatives contracts based on models that make use of historical information only, may hence result in substantial errors since the model leaves aside important, forward-looking information, although it is publicly available and likely to play a key role for individual trading decisions.

In this paper, we contribute to the literature by focusing on the prominent role of forward-looking information in electricity markets and investigating its impact on the pricing of electricity derivatives contracts. Based on an empirical analysis of 1-month and 2-months ahead forward contracts traded in the British market, we show that the integration of forecast data on fundamental variables, such as electricity demand or available capacity, significantly improves the performance of our electricity derivatives pricing model during those times when prices are highly sensitive to these drivers. By means of an enlargement-of-filtration approach, we demonstrate how to properly integrate forecasts of demand and capacity into our setting, and thus account for the apparent asymmetry between the historical filtration and the (enlarged) market filtration in electricity markets.

In general, existing literature on electricity spot price modeling can be grouped into two categories: on the one hand, often allowing for analytic derivatives pricing formulae, considerable attention has been devoted to reduced-form models that either directly specify dynamics for the electricity spot price process itself or, alternatively model the term structure of forward contracts, where spot dynamics are derived from a forward contract with immediate delivery (see, e.g., Clewlow and Strickland, 2000; Koekebakker and Ollmar, 2005; or Benth and Koekebakker, 2008). Starting with traditional commodity modeling approaches via mean-reverting one- or two-factor models (Lucia and Schwartz, 2002), the goal of reflecting the stylized facts of electricity spot price dynamics even more adequately has led to more elaborate settings including: regime-switching approaches (Janczura and Weron, 2010); affine jump diffusion processes (Bierbrauer et al., 2007); and settings with non-constant deterministic or stochastic jump intensities (Seifert and Uhrig-Homburg, 2007). However, for the purposes of our analysis, it will be important to employ a model that is capable, at least in some form, of reflecting the dependence structure between prices and the electricity markets' underlying drivers, which then allows us to also relate forward-looking information on these drivers to electricity prices. Hence, by their nature, classic reduced-form models appear unsuited in this context and will not be pursued in this paper. ${ }^{4}$

On the other hand, the class of structural/fundamental electricity price models subsumes a wide spectrum of more diverse modeling approaches; starting with equilibrium-based models (Bessembinder and Lemmon, 2002; Buehler and MuellerMehrbach, 2007; Aïd et al., 2011) or even more richly parameterized full production cost models (Eydeland and Wolyniec,

\footnotetext{
${ }^{1}$ See, e.g., Johnson and Barz (1999), Burger et al. (2004) or Fanone et al. (2013).

2 Benth and Meyer-Brandis (2009) provide several examples in support of this argument, such as the case of planned maintenance for a major generating unit, which is likely to be public information available to all market participants. Assuming a stylized setting, this outage will necessarily affect electricity spot prices expected to prevail during the time the unit is offline. Likewise, the outage will also affect today's prices of derivative contracts such as forward and futures contracts if their delivery periods overlap with the period of scheduled maintenance. However, in the absence of any means to economically store electricity bought at (cheaper) spot prices today and to sell it at higher prices during the time of the outage, there is no opportunity for arbitrage in such situation. This consequently implies that today's electricity spot prices will remain virtually unaffected by the announcement of the outage.

${ }^{3}$ In Europe, Regulations (EC) no. 1228/2003, its follow-up no. 714/2009, and annexed "Congestion Management Guidelines" (CMG) may serve as the most prominent example, requiring, e.g., that "the TSO shall publish the relevant information on forecast demand and on generation (...)" (CMG, article 5.7). In the US, similar standards are in place, e.g., as issued by the Federal Energy Regulatory Commission (FERC).

${ }^{4}$ Note that it is still possible to integrate information about the dynamics of fundamental state variables (such as demand or, e.g., also temperature) into reduced-form models by means of correlated processes. For an example, see Benth and Meyer-Brandis (2009). However, even though such models may bridge the gap between classic reduced-form and fundamental approaches, it is still questionable whether a single correlation parameter may be sufficient to reflect the rich dependence structures between electricity prices and a fundamental state variable - all the more if the dynamics of several underlying variables are to be taken into account at the same time.
} 
2002) on the one end, but also including, on the other end, econometric approaches such as regression-based settings (Karakatsani and Bunn, 2008) or time-series models whose efficiency is enhanced by including exogenous fundamental variables (Weron, 2006, or Misiorek et al., 2006).

Often referred to as hybrid approach, the class of models focused on in this study may be seen in the middle of this spectrum. In its most general form, fundamental settings of this kind comprise of a selection of separately modeled underlying factors, such as electricity demand, available generation capacity, and fuels. Along with a specification of the functional relationship between these factors and electricity spot prices, this setting can hence be interpreted as meritorder framework. ${ }^{5}$ The main challenge in this context is to be seen in an adequate reflection of the characteristic slope and curvature of the merit-order curve that is usually characterized by significant convexity. ${ }^{6}$ As a matter of simplification, many studies (see, e.g., Skantze et al., 2000; Cartea and Villaplana, 2008; or Lyle and Elliott, 2009) propose to approximate the merit-order curve with an exponential function. While there may be other functional specifications yielding a better fit, such as a piecewise defined "hockey stick" function (Kanamura and Ohashi, 2007) or power laws (Aïd et al., 2013), the exponential setting offers the key advantage of yielding log-normal electricity spot prices, allowing for analytic derivatives pricing formulae.

In order to provide timely pricing information to market participants by retaining tractability, our model also adopts an exponential setting for representing the merit-order curve. As regards the inclusion of generating fuels, we follow Pirrong and Jermakyan (2008) by modeling a stylized one-fuel market, leaving aside more flexible multi-fuel approaches (see Aïd et al., 2009, 2013; Coulon and Howison, 2009; Carmona et al., 2013). While our one-fuel setting avoids a model-endogenous determination of both the merit-order and the marginal fuel in place, it remains to be discussed how this reduction in flexibility affects pricing results, and for which markets such a simplification may be feasible at all.

Regarding the question of how to account for forward-looking information in this context, many of the above presented models could in fact be modified to accommodate short-, mid- or long-term forecasts about future levels of electricity demand or available capacity. However, previous literature mainly focuses on applying demand/capacity forecasts in a very short-term (day-ahead) context only (see, e.g., Karakatsani and Bunn, 2008; Bordignon et al., 2013). Other studies, instead, propose pricing models that also rely on forward-looking information, yet do not provide an exhaustive empirical analysis of how this affects pricing performance (see, e.g., Cartea et al., 2009; Burger et al., 2004).

Finally, the application of the enlargement-of-filtration approach to electricity markets was initially proposed by Benth and Meyer-Brandis (2009). Focusing on risk premia rather than on forward pricing, Benth et al. (2013) use this concept in order to analyze the impact of forward-looking information on the behavior of risk premia in the German electricity market. The authors develop a statistical test for the existence of an information premium ${ }^{7}$ and show that a significant part of the oftentimes supposedly irregular behavior of risk premia can be attributed to it. ${ }^{8}$

The remainder of this paper is structured as follows: in the next section, we develop our underlying electricity pricing model. Section 3 introduces the concept of the enlargement-of-filtration approach in the context of fundamental electricity price modeling. Section 4 starts with the empirical part by describing the data, the estimation methodology, as well as the general structure of the pricing study. Section 5 discusses the pricing results. Section 6 concludes.

\section{A fundamental electricity pricing model}

\subsection{Electricity demand}

Electricity demand is modeled on a daily basis with its functional specification chosen to reflect typical characteristics of electricity demand such as mean-reverting behavior, distinct seasonalities as well as intra-week patterns. On a filtered probability space $\left(\Omega, \mathcal{F}^{D}, \mathbb{F}^{D}=\left(\mathcal{F}^{D}\right)_{t \in\left[0, T^{\star}\right]}, \mathbb{P}\right)$ with natural filtration $\mathbb{F}^{q}=\left(\mathcal{F}^{q}\right)_{t \in\left[0, T^{\star}\right]}$ (for $\left.\mathcal{F}_{t}^{D}=\mathcal{F}_{0} \vee \mathcal{F}_{t}^{q}\right)$, demand $D_{t}$ is governed by the following dynamics:

$$
\begin{aligned}
& D_{t}=q_{t}+s^{D}(t), \\
& \mathrm{d} q_{t}=-\kappa^{D} q_{t} \mathrm{~d} t+\sigma^{D} \mathrm{e}^{\varphi(t)} \mathrm{d} B_{t}^{D},
\end{aligned}
$$

\footnotetext{
${ }^{5}$ Alternatively, this functional relationship can also be seen as inverse supply curve or bid-stack, if we abstract from generators submitting bids exceeding marginal costs. The merit-order setting is complemented by the assumption of electricity demand being completely inelastic, which is a basic assumption for models of this kind, and which is well in accordance with empirical realities. See Carmona and Coulon (2012) for further reference as well as for a general and comprehensive review of the fundamental modeling approach and its inherent assumptions.

${ }^{6}$ This is a non-trivial issue given that the curvature is determined by both the individual composition of generating units for each marketplace and their marginal cost structure which, in turn, depends stochastically on other factors such as underlying fuel prices, weather conditions, (un-)planned outages, and daily patterns of consumption. Additional factors may relate to market participants exercising market power by submitting strategic bids, but also regulatory regimes awarding, e.g., preferential feed-in tariffs to renewable energy producers.

${ }^{7}$ The information premium is defined as the difference between forward prices, depending on whether or not forward-looking information is entering the price formation mechanism.

${ }^{8}$ On a more general note, the idea to resort to forward-looking information, of course, extends to numerous other fields of academic research. Another "natural" candidate is, by way of example, the pricing of weather derivatives. For studies that resort to temperature forecasts in order to price temperature futures, see, e.g., Jewson and Caballero (2003), Dorfleitner and Wimmer (2010), and Ritter et al. (2011).
} 


$$
\begin{aligned}
& s^{D}(t)=a^{D}+b^{D} t+\sum_{i=2}^{12} c_{i}^{D} M_{i}(t)+c_{W E}^{D} W E(t)+\sum_{j=1}^{4} c_{P H_{j}}^{D} P H_{j}(t), \\
& \varphi(t=\theta \sin (2 \pi(k t+\zeta)),
\end{aligned}
$$

where $q_{t}$ is an OU-process with mean-reversion parameter $\kappa^{D}$ and a standard Brownian motion $B_{t}^{D}$. Since volatility of electricity demand has often been found to exhibit seasonal levels of variation (see, e.g., Cartea and Villaplana, 2008), ${ }^{9}$ we apply a time-varying volatility function as proposed by Geman and Nguyen (2005) or Back et al. (2013), with $\theta \geq 0$, a scaling parameter $k=\frac{1}{365}$, and $\zeta \in[-0.5 ; 0.5]$ to ensure uniqueness of parameters. In order to additionally reflect absolute-level demand-side seasonality, the deterministic component $s^{D}(t)$ contains monthly dummy variables $M_{i}(t)$ as well as indicators for weekends $W E(t)$ and public holidays. ${ }^{10} \mathrm{~A}$ linear trend is also included in $s^{D}(t)$ in order to capture the effect of structural developments in the respective market that may lead to an increase or decrease of electricity demand in the long term.

\subsection{Available capacity}

Available capacity $C_{t}$ is modeled in a similar manner as electricity demand. Hence, on a filtered probability space $\left(\Omega, \mathcal{F}^{C}, \mathbb{F}^{C}=\left(\mathcal{F}^{C}\right)_{t \in\left[0, T^{\star}\right]}, \mathbb{P}\right)$ with the natural filtration defined as $\mathbb{F}^{m}=\left(\mathcal{F}^{m}\right)_{t \in\left[0, T^{\star}\right]}$ (for $\mathcal{F}_{t}^{C}=\mathcal{F}_{0} \vee \mathcal{F}_{t}^{m}$ ), we specify the following dynamics:

$$
\begin{aligned}
& C_{t}=m_{t}+s^{C}(t), \\
& \mathrm{d} m_{t}=-\kappa^{C} m_{t} \mathrm{~d} t+\sigma^{C} \mathrm{~d} B_{t}^{C}, \\
& s^{C}(t)=a^{C}+b^{C} t+\sum_{i=2}^{12} c_{i}^{C} M_{i}(t)+c_{W E}^{C} W E(t)+\sum_{j=1}^{4} c_{P H_{j}}^{C} P H_{j}(t)+c_{R}^{C} R(t),
\end{aligned}
$$

where $m_{t}$ is again an OU-process with mean-reversion parameter $\kappa^{C}$. Volatility $\sigma^{C}$ is constant since, in contrast to demand $D_{t}$, available capacity $C_{t}$ is generally not found to exhibit seasonality in volatility levels. $B_{t}^{C}$ is a standard Brownian motion and $s^{C}(t)$ is defined equivalently to $s^{D}(t)$. In addition, another dummy variable $R(t)$ is included in order to reflect the fact that, unlike for the electricity demand data used in this study, capacity data relating to generation units from Scotland is available only after April 2005. ${ }^{11}$ Finally, in order to allow for potential correlation between electricity demand and available capacity, we define the parameter $\varrho_{C, D}$ as $\mathrm{d} B_{t}^{C} \mathrm{~d} B_{t}^{D}=\varrho_{C, D} \mathrm{~d} t$.

\subsection{Marginal fuel}

In addition to the processes for electricity demand and available capacity, we introduce the dynamics for our third state variable, i.e., the marginal fuel used for generation. For the sake of simplicity, we assume that the marginal fuel for the respective electricity market under study does not change. While this certainly is a restrictive assumption, it may still seem justified for markets that are strongly relying on one generating fuel only so that during baseload/peakload hours, spot markets are primarily cleared by plants that use the same fuel for generation. Reflecting the dominant role of natural gas as marginal generating fuel in the British market - and, more generally, in several other major electricity markets - we choose it as the single fuel to be included into our overall pricing model (see, e.g. Bunn and Fezzi, 2008 or, more generally, Blumsack, 2014).

Although for modeling natural gas, a variety of multi-factor approaches with varying degree of sophistication have been proposed by recent literature (see, e.g., Cartea and Williams, 2008, for an overview), we seek to limit both complexity and (the already high) parametrization of the model and, therefore, apply the mean-reverting one-factor model initially proposed by Schwartz (1997). On a given filtered probability space $\left(\Omega, \mathcal{F}^{g}, \mathbb{F}^{g}=\left(\mathcal{F}^{g}\right)_{t \in\left[0, T^{\star}\right]}, \mathbb{P}\right)$, the log gas price, ln $g_{t}$, is assumed to be governed by the following dynamics:

$$
\begin{aligned}
& \ln g_{t}=X_{t}+s^{g}(t), \\
& \mathrm{d} X_{t}=-\kappa^{g} X_{t} \mathrm{~d} t+\sigma^{g} \mathrm{~d} B_{t}^{g},
\end{aligned}
$$

\footnotetext{
${ }^{9}$ As our estimation results will show, volatility of electricity demand in the British market is higher during winter months than during summer months. However, this effect may be less pronounced or even reversed for other markets where, e.g., the need for air conditioning during summer months drives electricity demand to higher and more volatile levels than during winter months.

10 Since the extent of a demand reduction induced by a public holiday strongly depends on the respective season prevailing, three different groups of public holidays shall be distinguished: those occurring in winter $\left(\mathrm{PH}_{2}\right)$, the Easter holidays $\left(\mathrm{PH}_{3}\right)$, and others $\left(\mathrm{PH}_{4}\right)$. Additionally, the days with reduced electricity demand between Christmas and New Year are treated as quasi-public holidays $\left(\mathrm{PH}_{1}\right)$. This may appear overly detailed; however, almost all coefficients turn out to be highly significant. See Buehler Mueller-Mehrbach (2009) for an even more detailed approach.

11 The introduction of the British Electricity Trading and Transmission Agreements (BETTA) as per April 2005 is generally referred to as the starting point of a UK-wide electricity market. Prior to that, and although linked via interconnectors, the electricity markets of England/Wales and Scotland were operating independently.
} 


$$
s^{g}(t)=a^{g}+b^{g} t+\sum_{i=2}^{12} c_{i}^{g} M_{i}(t),
$$

where $X_{t}$ is the logarithm of the de-seasonalized price dynamics, $s^{g}(t)$ reflects the strong seasonality component that is inherent in natural gas prices, and $B_{t}^{g}$ is a standard Brownian motion. For completeness, parameters $\varrho_{g, D}$ and $\varrho_{g, C}$ are defined analogously as above, i.e. $\mathrm{d} B_{t}^{g} \mathrm{~d} B_{t}^{D}=\varrho_{g, D} \mathrm{~d} t$ and $\mathrm{d} B_{t}^{g} \mathrm{~d} B_{t}^{C}=\varrho_{g, C} \mathrm{~d} t$. Note that the overall structure of our power price model as well as the availability of closed-form solutions will be retained when introducing refinements such as a multi-factor lognormal model for natural gas. ${ }^{12}$

\subsection{Pricing model}

In order to link the three state variables - marginal fuel $g_{t}$, electricity demand $D_{t}$, and capacity $C_{t}-$ with electricity spot prices $P_{t}$, we employ an exponential setting, thus reflecting the convex relationship between prices and load/capacity as induced by the merit-order curve. At the same time, we assume power prices to be multiplicative in the marginal fuel. Both assumptions can be considered common practice and yield the following structural relationship between spot prices and state variables ${ }^{13}$

$$
P_{t}=\alpha g_{t}^{\delta} \mathrm{e}^{\beta D_{t}+\gamma C_{t}}
$$

or, in log-form

$$
\ln P_{t}=\ln \alpha+\delta \ln g_{t}+\beta D_{t}+\gamma C_{t},
$$

where $\delta$ can be interpreted as the elasticity of the electricity spot price with respect to changes in the natural gas price. Setting $\delta=1$ would thus allow to interpret $\mathrm{e}^{\beta D_{t}+\gamma C_{t}}$ as heat rate function. ${ }^{14}$ However, given that we primarily investigate baseload power prices in the empirical part of this paper, we acknowledge that the elasticity of baseload power prices with respect to natural gas may be varying and, hence, do not impose the restriction $\delta=1$.

Also, and as will be seen later, there is a subtle form of dependence between the parameters $\alpha$ and $\delta$. In order to give an intuition for the role of $\alpha$, and at the same time providing an abstract link to structural multi-fuel power price models, Eq (11) can be re-written as follows:

$$
P_{t}=\underbrace{f_{t}^{(1-\delta)}}_{\alpha} g_{t}^{\delta} \mathrm{e}^{\beta D_{t}+\gamma C_{t}} .
$$

In Eq. (13), $\alpha$ can hence be interpreted as reflecting the dynamics of another generating fuel $f_{t}$ (such as coal) which, however, will be held constant for simplicity.

Following classic theory, futures prices equal the expectation of the spot price at maturity under a suitably chosen riskneutral measure $\mathbb{Q}$ (Cox and Ross, 1976; Harrison and Kreps, 1979). However, the non-storability of electricity creates nonhedgeable risks, leading to an incomplete market setting. Therefore, the risk-neutral measure $\mathbb{Q}$ cannot be determined uniquely, but will instead be inferred from market prices of traded forward contracts, as will be shown in Section 4 . In order to govern the change of measure, and following Girsanov's theorem, we introduce separate market prices of risk $\lambda^{D}, \lambda^{C}$, and $\lambda^{g}$ for the different sources of uncertainty in our model. These market prices of demand, capacity, and fuel price risk are assumed constant. Given that $P_{t}$ is $\log$-normal in the state variables, the log futures price, $\ln F_{t}(T)$, at time $t$ with delivery date $T$ is given as follows:

$$
\begin{aligned}
& \ln F_{t}(T)=\mathbb{E}^{\mathbb{Q}}\left[\ln P_{T} \mid \mathcal{F}_{t}\right]+\frac{1}{2} \mathbb{V}^{\mathbb{Q}}\left[\ln P_{T} \mid \mathcal{F}_{t}\right] \\
& \ln F_{t}(T)=\ln \alpha+\delta \mathbb{E}^{\mathbb{Q}}\left[\ln g_{T} \mid \mathcal{F}_{t}\right]+\beta \mathbb{E}^{\mathbb{Q}}\left[D_{T} \mid \mathcal{F}_{t}\right]+\gamma \mathbb{E}^{\mathbb{Q}}\left[C_{T} \mid \mathcal{F}_{t}\right]+\frac{1}{2} \delta^{2} \mathbb{V} \mathbb{Q}\left[\ln g_{T} \mid \mathcal{F}_{t}\right]+\frac{1}{2} \beta^{2} \mathbb{V}^{\mathbb{Q}}\left[D_{T} \mid \mathcal{F}_{t}\right]+\frac{1}{2} \gamma^{2} \mathbb{V}^{\mathbb{Q}}\left[C_{T} \mid \mathcal{F}_{t}\right],
\end{aligned}
$$

\footnotetext{
12 Applying a one-factor model for natural gas prices may be seen as simplistic since the structure of this model implies that all natural gas forward/ futures contracts are perfectly correlated across maturities. However, note that we primarily focus on pricing short-term electricity forward contracts for which only the short end of the curve may be relevant. In contrast, when pricing longer-term electricity contracts, we suggest employing a two-factor natural gas price model instead.

${ }^{13}$ As mentioned in the overview of literature, note that - as is characteristic for this class of models - we thus derive electricity spot prices based on an exogenously given relationship between fuels, supply, and inelastic demand (see, e.g., Skantze et al., 2000; Cartea and Villaplana, 2008; Pirrong and Jermakyan, 2008; Lyle and Elliott, 2009 or, more generally, Carmona and Coulon, 2012; for the specific structure of Eq. (11), see Coulon et al., 2013 who follow a similar approach). Taking the dynamics for the spot as given, forward pricing formulae are then derived based on a no-arbitrage argument. Although a (possibly dynamic) equilibrium setting that explicitly models the optimization behavior of all participants in both spot and forward markets might provide additional insights, such as on the determinants of endogenously derived forward risk premia, we refrain from doing so here. Given that we primarily focus on the pricing impact of using forward-looking information, dynamic equilibrium settings might be unsuited for that purpose, e.g. due to a number of additionally required assumptions and/or unobserved variables, leading to calibration challenges, and implying reduced flexibility in general

${ }^{14}$ The heat rate indicates how many units of natural gas (or, more generally, of any other generating fuel) are required to produce one unit of electricity. In our case, the "market" heat rate would refer to the price-setting plant that generates the marginal unit of electricity.
} 
where $\mathbb{E}^{\mathbb{Q}}\left[\cdot \mid \mathcal{F}_{t}\right]$ and $\mathbb{V}^{\mathbb{Q}}\left[\cdot \mid \mathcal{F}_{t}\right]$ indicate expectation and variance under $\mathbb{Q}$ and conditional on $\mathcal{F}_{t}$ which is defined as $\mathcal{F}_{t}:=\mathcal{F}_{t}^{D} \vee \mathcal{F}_{t}^{C} \vee \mathcal{F}_{t}^{g} .{ }^{15}$ As further outlined in Section 3, when pricing forward contracts by making use of forecasts of electricity demand and capacity, forward prices will be computed as risk-neutral expectations of the spot price during the delivery period, conditional on $\mathcal{G}_{t}$ rather than $\mathcal{F}_{t}$. Consequently, Eq. (14) will need to be replaced by $\ln F_{t}(T)=\mathbb{E}^{\mathbb{Q}}\left[\ln P_{T} \mid \mathcal{G}_{t}\right]+\frac{1}{2} \mathbb{V}^{\mathbb{Q}}\left[\ln P_{T} \mid \mathcal{G}_{t}\right]$, where $\mathcal{G}_{t}:=\mathcal{G}_{t}^{D} \vee \mathcal{G}_{t}^{C} \vee \mathcal{F}_{t}^{g}$ and $\left(\mathcal{G}_{t}\right)_{t \in\left[0, T^{\star}\right]} \quad$ (or, more precisely, $\left(\mathcal{G}_{t}^{D}\right)_{t \in\left[0, T^{\star}\right]}$ and $\left.\left(\mathcal{G}_{t}^{\mathcal{C}}\right)_{t \in\left[0, T^{\star}\right]}\right)$ is the enlarged market filtration containing forecasts of expected demand and capacity levels, respectively.

Also note that Eq. (14) refers to a contract with delivery of electricity at some future date $T$, whereas standard electricity forward contracts specify the delivery of electricity throughout a delivery period $[T, \bar{T}]$ (with $T<\bar{T}$ ), e.g., one week or one month. Following Lucia and Schwartz (2002), we compute the price of a forward contract with delivery period $[T, \bar{T}]$, containing $n=\bar{T}-T$ delivery days, as the arithmetic average of a portfolio of $n$ single-day-delivery forward contracts with their maturities spanning the entire delivery period, i.e.

$$
F_{t}(\underline{T}, \bar{T})=\frac{1}{\bar{T}-\underline{T}} \sum_{i=1}^{n} F_{t}\left(\tau_{i}\right)
$$

Finally, calculating electricity forward prices based on Eq. (16) also requires us to have available the corresponding fuel forward prices with single-day maturities, i.e., one also needs to compute $\mathbb{E}^{\mathbb{Q}}\left[\ln g_{\tau_{i}} \mid \mathcal{F}_{t}^{g}\right]$ (as well as the conditional variance) for every day $\tau_{i}$ within the delivery period $[\underline{T}, \bar{T}]$. For that purpose, we take the log-spot price implied by the natural gas forward curve at time $t$ as a starting point to compute for every day $\tau_{i}$ within the delivery period the price of a hypothetical natural gas forward contract that matures on the same day. Hence, we calibrate to the gas curve for every pricing day before subsequently fitting the power forward curve. In this context, as a simplified approach, only one average value for $\mathbb{E}^{\mathbb{Q}}\left[\ln g_{\tau_{i}} \mid \mathcal{F}_{t}^{g}\right]$ during the entire delivery period could be used (e.g., based on the current value of the month-ahead natural gas forward, when pricing month-ahead electricity forwards). However, this may pose problems for non-standard delivery periods and would require identically defined delivery periods for gas and power. ${ }^{16}$

Having outlined our general modeling approach, a final comment on the modeling framework chosen may seem to be in order: in fact, given that our analysis concentrates on pricing electricity forward contracts, it would have been a natural choice to employ a reduced-form model that has the forward curve as a direct starting point, such as in Koekebakker and Ollmar (2005). However, we have refrained from doing so: first, although focusing on forward pricing, electricity spot prices will also be included into the estimation procedure of our model, which requires a framework that allows for an adequate reflection of the characteristic electricity (spot) price spikes. As shown by, e.g., Pirrong and Jermakyan (2008), the structural approach that we choose as the modeling framework in this paper is particularly suited for these purposes, which is not necessarily the case for standard direct models of the forward curve that "mechanically" deduct spot price dynamics from a forward contract with immediate maturity. In addition, while forward curve models typically rely on identifying underlying price drivers by means of a principal-components analysis (and subsequently extracting these hidden factors using the Kalman filter), we already have identified our underlying factors, such as demand and available capacity. Given the nonstorability of electricity, the respective levels of these factors observed today do not directly impact forward price levels; instead, they will only move forward prices if today's electricity demand is sufficiently high/low so that market participants believe such deviations from the long-run average will overlap with the delivery period of the respective forward contract. Hence, our 'hypothetic' model of the forward curve would involve modeling (conditional) expectations of demand and capacity, which is actually not too different from the modeling structure that we eventually yield by having the spot price as starting point for our model.

\section{The enlargement-of-filtration approach}

Non-storability of a given asset $Z$ implies that forward-looking information can neither be inferred from, nor is reflected in the historical evolution of its price trajectory $Z_{t}$ (Benth and Meyer-Brandis, 2009). Mathematically speaking, given a finite horizon $T^{\star}$ and letting $\left(\Omega, \mathcal{F}, \mathbb{F}=(\mathcal{F})_{t \in\left[0, T^{\star}\right]}, \mathbb{P}\right)$ be a filtered probability space, the natural filtration $\mathbb{F}^{Z}=\left(\mathcal{F}^{Z}\right)_{t \in\left[0, T^{\star}\right]}($ with $\mathcal{F}_{t}=\mathcal{F}_{0} \vee \mathcal{F}_{t}^{Z}$ ) may not reflect all forward-looking information available to market participants.

Assume that agents have access to some (non-perfect) forecast of the price of $Z$ at some future point in time $t^{\star} \in\left[0, T^{\star}\right]$. Then, there exists a sigma-algebra $\mathcal{G}_{\tau}$ with $\mathcal{F}_{\tau} \subset \mathcal{G}_{\tau}$ for all $\tau<t^{\star}$, where $\mathcal{G}_{\tau}$ reflects all available information including the forecast, whereas $\mathcal{F}_{\tau}$ does not. For $\tau \geq t^{\star}$, i.e. for times beyond the forecast horizon, we however have $\mathcal{F}_{\tau}=\mathcal{G}_{\tau}$, since no further forward-looking information is assumed to be available.

Next, note that whereas electricity clearly serves as most prominent example for non-storable underlyings, the above outlined incompleteness of natural filtrations with respect to forward-looking information can generally be extended to any kind of non-storable underlying. Therefore, and strictly speaking, we do not enlarge the filtration of the electricity spot price in order to incorporate forecasts, like Benth and Meyer-Brandis (2009) do in their reduced-form setting. Instead, we

\footnotetext{
${ }^{15}$ Note that the second part of Eq. (14) reflects our implicit assumption of all state variables being independent of each other, i.e., $Q_{C, D}=\varrho_{g, D}=\varrho_{g, C}=0$.

${ }^{16}$ Note, however, that in the UK, electricity forward contracts (still) trade according to the EFA (electricity forward agreement) calendar, grouping every calendar year into four quarters with three delivery months with lengths of $4 / 4 / 5$ calendar weeks, respectively. Consequently, delivery months of electricity forward contracts may not exactly overlap with corresponding delivery months of traded natural gas futures contracts.
} 
focus on electricity demand $D_{t}$ in Eq. (1) and available capacity $C_{t}$ in Eq. (5) which are, of course, non-storables as well, and hence do not reflect forward-looking information either. Therefore, and more precisely, it is the filtrations relating to the demand and capacity processes, respectively, that need to be enlarged in order to integrate forecasts provided by the system operator.

In the following, all formulae derived in this section relate to available capacity and forecasts thereof. For notational convenience, we work with de-seasonalized forecasts that relate to $m_{t}$ instead of $C_{t} ; \mathcal{F}_{t}^{C}$ and $\mathcal{G}_{t}^{C}$ are defined as further above. $^{17}$

In this setting, the (de-seasonalized) forecast of generation capacity available at time $t$ with forecast horizon $T$ is interpreted as $\mathcal{G}_{t}$-conditional expectation and expressed as

$$
\mathbb{E}^{\mathbb{P}}\left[m_{T} \mid \mathcal{G}_{t}^{C}\right]=m_{t} \mathrm{e}^{-\kappa^{C}(T-t)}+\sigma^{C} \mathbb{E}^{\mathbb{P}}\left[\int_{t}^{T} \mathrm{e}^{-\kappa^{C}(T-u)} \mathrm{d} B_{u}^{C} \mid \mathcal{G}_{t}^{C}\right] .
$$

This raises the question of how to treat expectations like $\mathbb{E}^{\mathbb{P}}\left[\int_{t}^{T} \mathrm{e}^{-\kappa^{C}(T-u)} \mathrm{d} B_{u}^{C} \mid \mathcal{G}_{t}^{C}\right]$ that are conditional on $\mathcal{G}_{t}^{C}$ (i.e., the sigmaalgebra including forecasts) when $B_{t}^{C}$, however, is an $\mathcal{F}_{t}^{C}$-adapted Brownian motion. Consequently, $B_{t}^{C}$ may no longer be a standard Brownian motion with respect to $\left(\mathcal{G}_{t}^{C}\right)_{t \in\left[0, T^{\star}\right]}$. Even more importantly, and following the "average approach" in Eq. (16), the pricing of, e.g., a forward contract with delivery period of one month will require us to ideally have capacity forecasts for every day within the delivery period. Yet, as is outlined in Section 4, detailed forecasts on a daily basis, as released by National Grid for the British market, only cover a window of the next 14 days. For longer-term prognoses, such as expected available capacity in 21 days, only forecasts of weekly granularity are published. Consequently, we may at best cover a certain first part of the delivery period with daily forecasts, whereas for the rest of the period, only a few weekly forecast points will be available, thus leaving several delivery days "uncovered" by forecasts. Hence, another key question is how to consistently determine $\mathbb{E}^{\mathbb{P}}\left[m_{T} \mid \mathcal{G}_{t}^{C}\right]$ when forecasts for capacity on delivery day $T$ are not available, but only for times $T_{1}$ and $T_{2}$ with $T_{1} \leq T \leq T_{2}$. This leads to the below considerations, which follow directly from applying Propositions 3.5 and 3.6 in Benth and Meyer-Brandis (2009) to our framework. Detailed derivations for the more general case of non-constant deterministic volatility are provided in the technical Appendix A.

Assume market participants are provided with forecasts of available capacity at future points in time $T_{1}$ and $T_{2}$, i.e., $\mathbb{E}^{\mathbb{P}}\left[m_{T_{1}} \mid \mathcal{G}_{t}^{C}\right]$ and $\mathbb{E}^{\mathbb{P}}\left[m_{T_{2}} \mid \mathcal{G}_{t}^{C}\right]$. Then, for $t \leq T_{1} \leq T \leq T_{2}$, capacity expected to be available at time $T$ can be derived as

$$
\mathbb{E}^{\mathbb{P}}\left[m_{T} \mid \mathcal{G}_{t}^{C}\right]=\mathbb{E}^{\mathbb{P}}\left[m_{T_{1}} \mid \mathcal{G}_{t}^{C}\right] \mathrm{e}^{-\kappa^{C}\left(T-T_{1}\right)}+\mathbb{E}^{\mathbb{P}}\left[\int_{T_{1}}^{T_{2}} \mathrm{e}^{\kappa^{C} u} \mathrm{~d} B_{u}^{C} \mid \mathcal{G}_{t}^{C}\right] \sigma^{C} \frac{\mathrm{e}^{\kappa^{C}} T\left(1-\mathrm{e}^{-2 \kappa^{C}\left(T-T_{1}\right)}\right)}{\mathrm{e}^{2 \kappa^{C} T_{2}}-\mathrm{e}^{2 \kappa^{C} T_{1}}} .
$$

The first part of the second term on the RHS of Eq. (18) is given as follows:

$$
\mathbb{E}^{\mathbb{P}}\left[\int_{T_{1}}^{T_{2}} \mathrm{e}^{\kappa^{C} u} \mathrm{~d} B_{u}^{C} \mid \mathcal{G}_{t}^{C}\right]=\frac{1}{\sigma^{C}}\left(\mathbb{E}^{\mathbb{P}}\left[m_{T_{2}} \mid \mathcal{G}_{t}^{C}\right] \mathrm{e}^{\kappa^{C} T_{2}}-\mathbb{E}^{\mathbb{P}}\left[m_{T_{1}} \mid \mathcal{G}_{t}^{C}\right] \mathrm{e}^{\kappa^{C} T_{1}}\right) .
$$

Note that we do not impose any specific structure on the nature of the enlarged filtration $\left(\mathcal{G}_{t}^{C}\right)_{t \in\left[0, T^{\star}\right]}$ apart from the facts that (i) the forecasts released by the TSO are interpreted as $\mathcal{G}_{t}$-conditional expectations and (ii) the $\mathcal{F}_{t}$-adapted process $B_{t}^{C}$ (likewise $B_{t}^{D}$ ) is a semi-martingale under the enlarged filtration. The latter is a common and well-studied approach in the enlargement-of-filtration theory, although more recent studies (Biagini and Oksendal, 2005; Di Nunno et al., 2006) have shown that such assumption could in fact be relaxed. As is shown in the appendix in more detail, the general idea in this case is that $B_{t}^{C}$ under the enlarged filtration $\left(\mathcal{G}_{t}^{C}\right)_{t \in\left[0, T^{\star}\right]}$ decomposes into a standard Brownian motion $\hat{B}_{t}^{C}$ and a drift term $A(t)=\int^{t} \vartheta(s) \mathrm{d} s$ which is usually referred to as the information drift. Hence, the additional information is essentially incorporated in the drift term $\vartheta(t)$, so that the dynamics for $m_{t}$ in Eq. (6) can be re-written as follows:

$$
\mathrm{d} m_{t}=-\kappa^{C}\left(m_{t}-\frac{\sigma^{C}}{\kappa^{C}} \vartheta(t)\right) \mathrm{d} t+\sigma^{C} \mathrm{~d} \hat{B}_{t}^{C} .
$$

Based on Eq. (20) - or, equivalently, on Eq. (18) - we can now compute $\mathcal{G}_{t}$-conditional expectations which relate to those points in time where no TSO forecasts are available, but which are still consistent with the modified stochastic dynamics as imposed by the available forecast points. Although a related concept, the change of the drift for the above capacity process has not been obtained through a change of the probability measure, i.e., $\hat{B}_{t}^{C}$ is a $\mathcal{G}_{t}$-adapted Brownian motion under the statistical measure $\mathbb{P}$. Therefore, when it comes to derivatives pricing under a risk-neutral measure $\mathbb{Q}$ in Section 5 , we consequently look for a $\mathcal{G}_{t}$-adapted standard $\mathbb{Q}$-Brownian motion $\breve{B}_{t}^{C}=\hat{B}_{t}^{C}-\Lambda_{\mathcal{G}}^{C}(t)$, where $\Lambda_{\mathcal{G}}^{C}(t)$ is a finite variation process representing the market price of risk that will be inferred from prices of electricity derivative contracts.

\footnotetext{
17 One could argue that there exist, of course, numerous other forecasts about expected available capacity that market participants might also have access to. E.g., capacity forecasts released by the system operator that relate to intermittent energy sources, such as wind or solar power, might be adjusted based on a utility's proprietary model involving different meteorological assumptions, e.g., more windy conditions or fewer sunshine hours. Likewise, the same is true for demand forecasts if market participants expect, e.g., higher temperatures than implied by the forecast of the system operator. Therefore, if we speak of $\mathcal{G}$ as the sigma-algebra "including forecasts", we assume away the existence of other forecasts and only mean to refer to those forecasts released by the TSO.
} 
Finally, we briefly discuss why we propose to use this specific approach of integrating demand and capacity forecasts here. In fact, one may think of alternative ways of how the incorporation of forward-looking information could be dealt with.

Assuming the forecast data to be reasonably reliable, one approach would be to interpret the forecasts as being released under perfect foresight and, hence, treating $D_{t}$ and $C_{t}$ as deterministic processes. In such case, demand and capacity forecasts, ultimately represented by expected values in Eq. (14), would be replaced by constants, so that the corresponding variance terms vanish. Although appealing by its simplicity, this approach raises several issues: first, when pricing, e.g., a forward contract with monthly delivery period, it is often the case that detailed forecast data on a daily basis is not available for all days of the delivery month. Especially for mid- to long-term forecasts, granularity of forecast points tends to be rather low, i.e., only expected maximum weekly, monthly, or seasonal demand (capacity) levels may be indicated. Irrespective of the question of whether long-term forecasts are still sufficiently accurate at all in order to justifiably treat them as deterministic, the necessary interpolation of missing long-term forecasts will induce some kind of arbitrariness. Given a variety of different interpolation methods to choose from, pricing results would consequently be quite sensitive to the specific technique selected. Second, and as is analyzed further below, future capacity levels are generally known to be hard to predict, in particular for the British market (see, e.g., Karakatsani and Bunn, 2008). This results in slightly less reliable forecasts, hence invalidating the assumption of deterministic forecasts in the first place, and leading to increased modeling risk otherwise.

A related approach has been presented by Ritter et al. (2011) and Haerdle et al. (2012) in the context of weather derivatives pricing. In case of missing daily forecast points for periods beyond the horizon of the daily forecasts, they propose to proceed as follows: the respective stochastic process is estimated based on a time series of historical data that has been extended to also include a given set of available daily forecasts, treating the latter as if they were actually observed. Missing forecasts are then replaced with expectations derived from the estimated process. Generally speaking, estimating parameters based on historic and forecast data at the same time may come close to the general idea of enlarging the information filtration. However, implementing this approach again comes at the cost of having to consider daily forecasts as deterministic whenever available. In addition, it is not a priori clear how to implement the "combined" estimation strategy when estimating a process on a daily basis and forecasts are given, e.g., on a weekly basis only, yet shall nevertheless be included in the estimation procedure. Importantly, parameter estimates, such as the speed of mean-reversion, may be critically affected, especially when during the estimation procedure, more weight is given to the forecast data relative to the realized data. Note that this issue is avoided by modifying demand and capacity dynamics as proposed in Eq. (20), while retaining the basic $\mathcal{F}$-implied stochastic properties of the respective processes at the same time.

Finally, as a third approach that might be appealing to practitioners, techniques similar to yield curve calibration in fixed income could be used: while the naive approach of incorporating demand forecasts directly into the seasonal function $s^{D}(T)$ is wrong, ${ }^{18}$ it is possible to re-fit the mean-reversion level, and let $s^{D}(T)$ adjust, so that expectations will correctly match the forecast points. For forecast data with high granularity (e.g., daily), results will be similar to the enlargement-of-filtration approach, but additional assumptions for interpolation will be necessary for the case of more widely spaced forecast points, as well as on which functional representation to use for $s^{D}(T)$ when expectations beyond the forecast horizon are to be computed.

\section{Data and estimation approach}

\subsection{Fundamental data}

The data set used in this study for the fundamental variables demand and capacity comprises of 10 years of historical data for the British electricity market, covering the period from 2002 up to 2011. These contain both historical realized and historical forecast data, and were obtained from National Grid, the British TSO, and Elexon, the operator of the balancing and settlement activities in the British market. ${ }^{19}$ Fig. 1 shows the development of the realized demand and capacity data during the period from 1 January 2007 to 31 December 2011, i.e., the period covered by our pricing study, whereas the prior five years are used as estimation period.

With respect to electricity demand, realized data is based on the outturn average megawatt (MW) value of electricity demand in England, Wales, and Scotland during the peak half-hour of the day, as indicated by operational metering. Specifically, we use the demand metric IO14 DEM which includes transmission losses and station transformer load, but excludes pump storage demand and net demand from interconnector imports/exports. ${ }^{20}$

Forecasts of expected electricity demand can be classified into two categories: first, National Grid releases daily updated demand forecasts covering the next 2 weeks ahead with daily granularity. These are forecasts of electricity demand

\footnotetext{
${ }^{18}$ Note that this will distort the relationship between the speed of mean-reversion of the OU-component and the actual level of mean-reversion, as imposed by the parameter estimates determined from historical data.

${ }^{19}$ National Grid both owns and operates the systems in England and Wales. Since the start of BETTA in April 2005, it has also been operating the highvoltage networks in Scotland owned by Scottish and Southern Energy as well as Scottish Power. The following websites were accessed: http://www. nationalgrid.com, http://www.bmreports.com, and http://www.elexonportal.co.uk.

${ }^{20}$ The British electricity market is connected to neighboring markets via interconnectors such as to/from France (IFA), the Netherlands (BritNed), or the Moyle Interconnector (connection to Northern Ireland).
} 

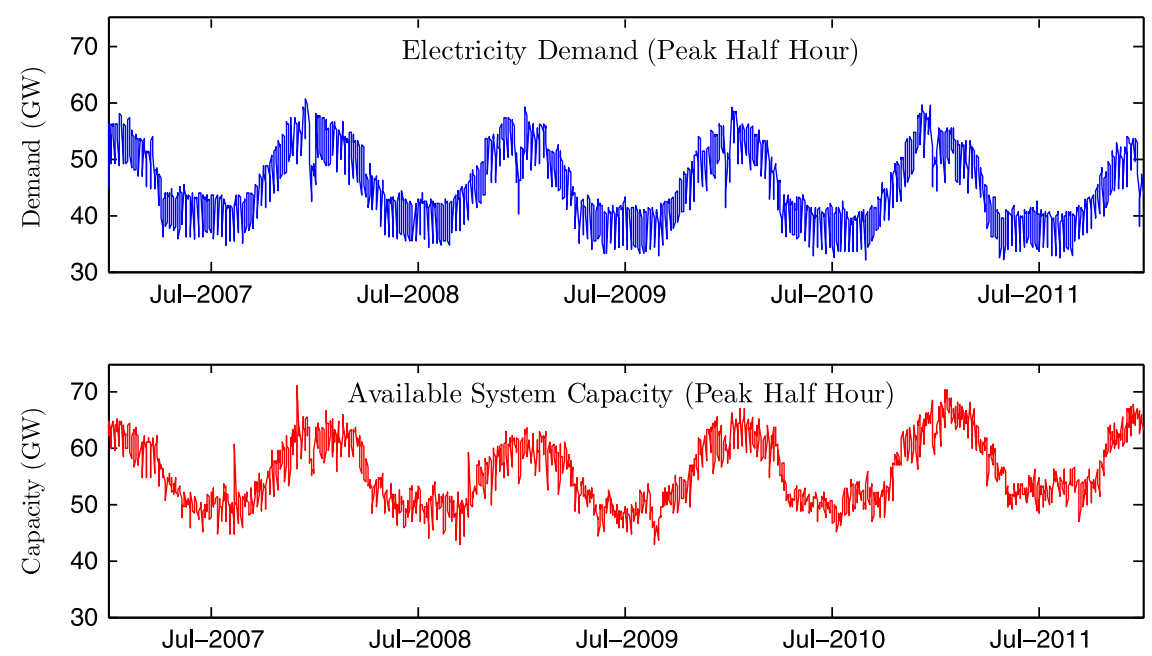

Fig. 1. Daily electricity demand and available system capacity. This figure shows the time series of realized daily electricity demand and available system capacity in the British market during the period from 1 January 2007 to 31 December 2011. Displayed demand and capacity data both relate to the same daily peak (demand) half hour. All data shown were obtained from National Grid and Elexon.

expected to prevail during the peak half-hour of the respective day, which is the reason why we use peak demand instead of average baseload demand throughout this study. Second, longer-term forecasts of expected demand are released once a week, covering the next 2-52 weeks ahead with weekly granularity. These forecasts relate to expected demand during the peak half-hour of the respective week. Fig. 2 provides a schematic overview of the different forecast horizons in the context of pricing a forward contract with monthly delivery period.

In terms of realized capacity available, National Grid records maximum export limits (MEL) for each of the units that are part of the overall balancing mechanism (BM). ${ }^{21}$ These limits quantify the maximum power export level of a certain BM unit at a certain time and are indicated by generators to the TSO prior to gate closure for each settlement period. ${ }^{22}$ In case of an (un-)expected outage for some generation unit, generators will accordingly submit a MEL of zero during the time of the outage for this unit. Moreover, since MEL do include volatile interconnector flows as well as anticipated generation from intermittent/renewable sources, they can be seen as a good real-time proxy of available generation capacity that either is in use for production, or could additionally be dispatched into the transmission system immediately.

The capacity forecasts used in our study are released by National Grid, too, and specifically relate to the forecasts of expected total generation availability of plants that are part of the balancing mechanism. Note that the data was adjusted for a few minor items in order to have in place consistently defined realized and forecast capacity levels that structurally are based on the same underlying reporting units. ${ }^{23}$ Both the timescale and updating structure of these forecasts are similar to the demand case.

When feeding the forecast data into our model, note that the weekly updated demand forecasts with a forecast horizon ranging from 2 to 52 weeks ahead are specified in correspondence to the expected peak half-hour within the respective week, i.e., it is not tied to a specific business day. Weekly capacity forecasts then relate to this very same half hour of expected peak demand, but do not specify an exact date either, which, however, is required in order to apply Eq. (18). Based on historic data, the peak half-hour of demand during a given week was most often found to occur between Tuesday and Thursday. For the sake of simplicity, we hence assume that weekly demand and capacity forecasts always refer to the Wednesday of the respective week. ${ }^{24}$

Finally, an important caveat applies: while forward-looking information may presumably be beneficial for derivatives pricing, outdated forward-looking information may certainly lead to the opposite. In fact, depending on both maturity and length of the delivery period for the respective contract to be priced, it may be the case that $\mathbb{E}^{\mathbb{P}}\left[D_{\tau} \mid \mathcal{G}_{t}^{D}\right]$ and $\mathbb{E}^{\mathbb{P}}\left[C_{\tau} \mid \mathcal{G}_{t}^{C}\right]$ for

\footnotetext{
21 These are approximately 300 units in Great Britain, with one plant comprising several units.

22 In the British market, gate closure is set at one hour before each half-hourly trading period. It refers to that point in time by when all market participants have to give notice about their intended physical positions so that the TSO can take action to balance the market.

${ }^{23}$ When using (unadjusted) generation availability forecasts, and unlike for the case of demand data, capacity forecasts still slightly differ in definition from the capacity metric on which the realized data is based (i.e., MEL). There are several reasons for this: Inter alia, volatile interconnector flows are hard to predict and, hence, are set at float throughout all forecast horizons. Also, a small number of generating units submit a MEL which, however, is not included into the forecast of generation capacity. We roughly adjust for these items to still arrive at consistently defined metrics, e.g., by carrying over latest observed values/forecast deviations into the future. At the same time, special focus is laid on our adjustments to remain simple, easily reproducible, and hence likely to be used by market participants. Further details are available from the authors upon request.

24 Pricing errors have proven to be rather insensitive to this assumption, i.e., fixing the weekly forecasts to relate to each Tuesday or Thursday of a given week (or even alternating, based on the business day for which the weekly peak-hour during the preceding week was observed) did not visibly change results.
} 


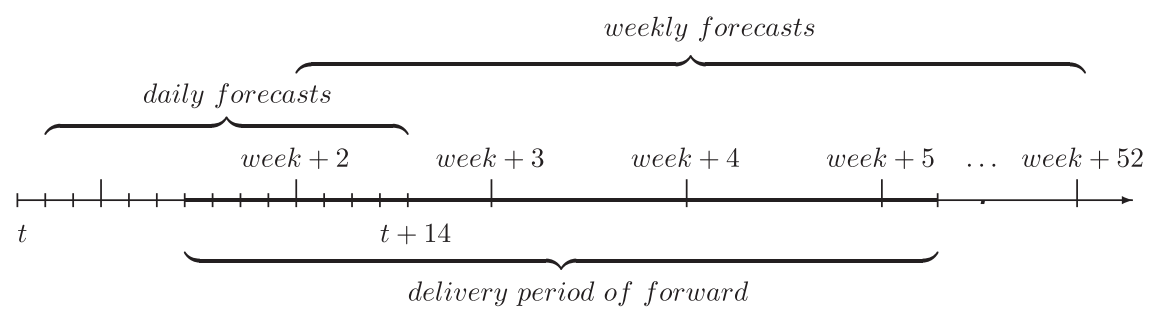

Fig. 2. Schematic overview of forecast horizons for the GB market. Daily forecasts are available on a 2- to 14-days-ahead basis; additionally, forecasts of expected maximum demand (capacity) per week are released for weeks 2-52. In this example, the first nine delivery days of some given forward contract are covered by daily forecasts, expected demand and capacity for each of the remaining days must be derived based on Eqs. (18) and (37).

$\tau=T \ldots \bar{T}$ are exclusively determined based on longer-term forecast points which are only updated weekly, as opposed to the daily updated 2-14 day-ahead forecasts. Focusing specifically on capacity forecasts, it may, however, happen that a major unplanned outage occurs just after the most recent weekly forecasts have been released. Even worse, for few periods in our data sample, forecast updates are missing altogether, leaving gaps of up to several weeks between successive forecast updates. Including such outdated forecasts into our (or any other) model and not updating for significant outages (whenever indicated) that move the market, hence unduly punishes the forecast-based model. Therefore, in case of missing updates or major unplanned outages not reflected in the most recent set of capacity forecasts, we have adjusted for such events by combining the forecast data with information provided on Bloomberg's "UK VOLTOUT" page as well as in news reports from ICIS Heren. Note that this information was available to market participants at the time of trading. ${ }^{25}$

\subsection{Electricity spot and forward data}

Following the historic development of electricity market regulation and especially since the inception of the New Electricity Trading Arrangements (NETA) regime in 2001, wholesale trading in the British market is predominantly characterized by OTC forward transactions with physical settlement. The forward market, defined as covering maturities from day-ahead up to several years ahead of delivery, makes up for about $90 \%$ of overall electricity volume traded in the UK (Wilson et al., 2011). Compared to other major European electricity markets such as Germany or the Nordic market, financially-settled trades are less common and mainly concentrate on limited exchange-based trading activity such as at the Intercontinental Exchange or at the APX UK exchange. More recently, the N2EX platform, operated by Nord Pool Spot and Nasdaq OMX Commodities and established in order to re-strengthen exchange-based trading, has also started to list cashsettled power futures contracts for the British market. Despite these developments, exchange-based derivatives trading activity still seems to be rather limited, with member participation in futures trading increasing at slow pace only (see OFGEM, 2011).

In view of this dispersed market structure with the vast majority of trades still being bilateral or broker-based, our electricity price data is exclusively based on OTC contracts and was obtained from two sources: first, Bloomberg provides historical forward prices which are defined as composite quotes from a panel of OTC brokers. Second, we obtained a comprehensive data set from Marex Spectron, a leading independent energy broker that operates one of Europe's largest and most established marketplaces for electricity. This second data set is entirely based on trade data (including time stamp of trade, executed through platform or voice brokers) and contains a variety of additional types of electricity contracts, out of which a second OTC sample was formed. These two samples, for which pricing errors are analyzed separately in Section 5, contain the following types of contracts: the "Bloomberg Data Set" comprises of 1-month ahead forward contracts; the "Marex Spectron Data Set" contains both 1-month and 2-months ahead forward contracts.

All selected forward contracts are baseload contracts. Moreover, electricity spot (i.e., day-ahead) price data is additionally used for model calibration purposes, but is not analyzed further in the main study. We deliberately focus on pricing the above types of baseload contracts, leaving aside other instruments with quarterly, seasonal, or yearly delivery periods. This is due to the following reasons: first, we are primarily interested in the pricing impact when considering demand and capacity forecasts, compared to a situation when disregarding such forecasts. Since these forecasts are more accurate for short-term horizons, ${ }^{26}$ our study focuses on contracts with short maturities and delivery periods. Second, trading activity generally concentrates on front months with liquidity at the longer end of the curve rapidly decreasing (OFGEM, 2011). Finally, and again primarily for liquidity reasons, we have chosen to analyze baseload contracts instead of peakload contracts. The fact that we are pricing baseload contracts, although using demand and capacity during peak half-hours as inputs, may seem inconsistent, but is ultimately due to the forecast data being available in this format only. It might be

\footnotetext{
${ }^{25}$ Prominent examples, amongst others, relate to several of the unplanned trippings of nuclear generation units during 2007/2008, which along with increased retrofitting activities of coal-based plants at that time led to extremely tight levels of available capacity in the British market.

${ }^{26}$ Longer-term forecasts rely on statistical averages and, thus, should convey no significant additional information as compared to the "no-forecast" case that is characterized by filtration $\left(\mathcal{F}_{t}\right)_{t \in\left[0, T^{\star}\right]}$.
} 
possible to convert the peakload demand and capacity forecasts into corresponding baseload predictions, e.g., by applying scaling factors that are based on historical averages. However, this is already indirectly accounted for by the estimation procedure outlined in the following subsection.

An overview of the two data samples is provided in Table 1 where descriptive statistics as well as further contractual characteristics for the day-ahead and forward contracts are summarized. As can be seen, the data exhibits well-known characteristics of electricity prices, such as substantial levels of volatility and excess kurtosis. While generally these effects are more pronounced for spot than for forward contracts, we also note the obvious difference in skewness of log-returns between both types of contracts.

\subsection{Estimation approach}

The individual processes for the state variables demand $\left(D_{t}\right)$ and available capacity $\left(C_{t}\right)$ are estimated by discretizing Eqs. (2) and (6) and using maximum likelihood. Based on annually rolling windows of five years of historical realized time series data, parameters are re-estimated annually, but held constant throughout every subsequent year when used for pricing purposes. This step of the estimation approach is based on realized data only, and no forecast data is used at this stage, irrespective of whether or not our derivatives pricing model makes use of forward-looking information in the end. ${ }^{27}$

Given the already very high number of parameters to be estimated, we have chosen a rather simple one-factor approach to model the dynamics of the marginal fuel used for generation, i.e., natural gas in our case. Since the spot component $X_{t}$ in Eq. (8) cannot be observed directly, estimation of all parameters for the natural gas model is instead performed based on futures data, by using the Kalman filter and maximum likelihood. Reformulating the model into state-space representation with corresponding transition and measurement equations is a standard exercise (see, e.g., Schwartz, 1997). Since our study primarily focuses on the pricing of short-term electricity forward contracts, we refer to the short end of the natural gas curve and, hence, seek to infer the log-spot natural gas dynamics from corresponding short-term futures contracts with maturities ranging from one to four months. Relevant data is sourced from Bloomberg and relates to natural gas futures contracts traded at the Intercontinental Exchange (ICE) with physical delivery at the National Balancing Point (NBP), the virtual trading hub for natural gas in Great Britain.

Having estimated the parameters that govern the dynamics of the respective underlying variables $D_{t}$, $C_{t}$, and $g_{t}$, the parameters $\alpha, \beta, \gamma$, and $\delta$ that link the three fundamental factors yet remain to be determined. Generally, two approaches appear suitable: first, based on Eq. (12), historic log electricity spot prices, $\ln P_{t}$, are regressed on corresponding time series data of $D_{t}, C_{t}$, and $\ln g_{t}$. This approach is proposed by Cartea and Villaplana (2008) for a structurally similar model that, however, does not include marginal fuel dynamics or forward-looking information. Second, implicitly (re-)estimating the parameters over time, based on a cross-section of electricity spot and forward prices.

Given evidence that $\alpha, \beta, \gamma$, and $\delta$ may not be constant over time, we favor the second approach. For instance, Karakatsani and Bunn (2008) also apply fundamentals-based models in their study on electricity spot price forecasting in the British market. They conclude that the models with the best pricing performance are those that allow for time-varying coefficients to link the fundamental factors. Moreover, the changing level of dependence of electricity spot prices on each fuel price due to mixing of bids and merit order changes as proposed in the model by Carmona et al. (2013) may be seen in the same spirit. Therefore, and although treated as constants in our model, the time-varying nature of the parameters $\alpha, \beta, \gamma$, and $\delta$ is captured by implicitly extracting and re-estimating them weekly from the cross-section of quoted power prices. Likewise, the parameters $\lambda^{D}$ and $\lambda^{C}$ governing the change of measure from $\mathbb{P}$ to $\mathbb{Q}$ are inferred in the same way.

In order to implicitly estimate these parameters, the following objective function is minimized:

$$
\begin{aligned}
\Phi_{W}^{\star} & =\underset{\Phi_{W}}{\arg \min } \operatorname{RMSPE}\left(\Phi_{W}\right) \\
& =\underset{\Phi_{W}}{\arg \min }\left[\sqrt{\frac{1}{N_{W}^{P}} \sum_{i=1}^{N_{W}^{P}}\left(\frac{\hat{P}_{W, i}\left(\Phi_{W}^{\mathbb{P}}\right)-P_{W, i}}{P_{W, i}}\right)^{2}}+\sqrt{\frac{1}{N_{W}^{F}} \sum_{i=1}^{N_{W}^{F}}\left(\frac{\hat{F}_{W, i}\left(\Phi_{W}^{\mathbb{Q}}\right)-F_{W, i}}{F_{W, i}}\right)^{2}}\right],
\end{aligned}
$$

where $\Phi_{W} \equiv\left\{\alpha, \beta, \gamma, \delta, \lambda^{D}, \lambda^{C}\right\}$ with the two subsets $\Phi_{W}^{\mathbb{Q}}$ and $\Phi_{W}^{\mathbb{P}}$ defined as $\Phi_{W} \equiv \Phi_{W}^{\mathbb{Q}}$ and $\Phi_{W}^{\mathbb{P}} \equiv \Phi_{W}^{\mathbb{Q}} \backslash\left\{\lambda^{D}, \lambda^{C}\right\}$. To minimize the root mean squared percentage error (RMSPE) over the in-sample period $W$, we assemble all available day-ahead prices $P_{W, i}$ (totaling $N_{W}^{P}$ quotes) as well as all available forward prices $F_{W, i}$ ( $N_{W}^{F}$ quotes) and compare against prices $\hat{P}_{W, i}$ and $\hat{F}_{W, i}$ as predicted by our model based on Eqs. (12) and (14). For in-sample estimation windows $W$, we use a length of eight weeks, e.g., $w_{1}-w_{8}$, for the Bloomberg sample. Out-of-sample testing of the model is performed during the subsequent week (i.e., $w_{9}$ ), employing the parameters estimated over $W$ - thus only using information available up to the respective pricing day. Finally, the in-sample period is shifted by one week (i.e., new window: $w_{2}-w_{9}$ ) and parameters are re-estimated. For the Marex Spectron sample, we shorten the length of the in-sample estimation windows to six weeks since more price observations per week are available, thus allowing for a robust estimation with a shorter window. Furthermore, these changes in the in-sample set-up may provide additional robustness to our findings examined in Section 5, so as to ensure that pricing improvements from using forecasts do not rely on a specific mix of contracts or length of in-sample estimation windows. With these settings in place, the eight-week in-sample window $W$ used for the Bloomberg sample comprises approx. 38 forward prices and

${ }^{27}$ Also, as regards the above mentioned problem of missing updates or major planned outages, note that this problem relates to forecast data only, yet not to the realized outturn capacity data which we use at this step of the estimation procedure. 
Table 1

Samples of baseload spot and forward contracts. This table reports summary statistics for the samples of electricity spot (day-ahead) and forward prices covering the period from 2 January 2007 until 30 December 2011. $[\underline{T}, \bar{T}]$ denotes the average delivery period (in days) and $T-t$ the average maturity (in days) as measured until the start of the delivery period. All contracts from both the Bloomberg and Marex Spectron samples are baseload contracts. Displayed log-returns for 1- and 2-month(s) ahead forward contracts are adjusted to account for roll-over of contracts as well as for missing quotes.

\begin{tabular}{|c|c|c|c|c|c|c|c|}
\hline Data & Mean & Median & Std. dev. & Skewness & Kurtosis & {$[\underline{T}, \bar{T}]$} & $\underline{T}-t$ \\
\hline \multicolumn{8}{|l|}{$\begin{array}{l}\text { Bloomberg data } \\
\text { 1-Day ahead }\end{array}$} \\
\hline $\ln P t$ & 3.7543 & 3.7600 & 0.3891 & 0.0818 & -0.1121 & 1.0 & 1.0 \\
\hline $\begin{array}{l}\ln P_{t}-\ln P_{t-1} \\
\text { 1-Month ahead }\end{array}$ & -0.0019 & -0.0019 & 0.0717 & 1.2812 & 12.8443 & & \\
\hline $\operatorname{lnFt}$ & 3.7781 & 3.7899 & 0.3737 & 0.2196 & 0.2131 & 30.4 & 15.9 \\
\hline $\begin{array}{l}\ln F_{t}-\ln F_{t-1} \\
\text { Marex Spectron data } \\
\text { 1-Day shead }\end{array}$ & -0.0009 & -0.0003 & 0.0219 & -0.2365 & 5.4731 & & \\
\hline $\ln \mathrm{Pt}$ & 3.7500 & 3.7612 & 0.3829 & 0.1225 & 0.0516 & 1.0 & 1.0 \\
\hline $\begin{array}{l}\ln P_{t}-\ln P_{t-1} \\
\text { 1-Month shead }\end{array}$ & -0.0027 & -0.0021 & 0.0721 & 1.2138 & 10.9920 & & \\
\hline $\ln F t$ & 3.7856 & 3.7956 & 0.3604 & 0.2359 & 0.4641 & 30.4 & 16.1 \\
\hline $\begin{array}{l}\ln F_{t}-\ln F_{t-1} \\
\text { 2-Months shead }\end{array}$ & -0.0010 & -0.0014 & 0.0205 & -0.1641 & 4.8328 & & \\
\hline $\operatorname{lnFt}$ & 3.8003 & 3.7975 & 0.3532 & 0.3031 & 0.4328 & 30.8 & 44.8 \\
\hline $\ln F_{t}-\ln F_{t-1}$ & -0.0009 & -0.0005 & 0.0177 & -0.3552 & 4.5943 & & \\
\hline
\end{tabular}

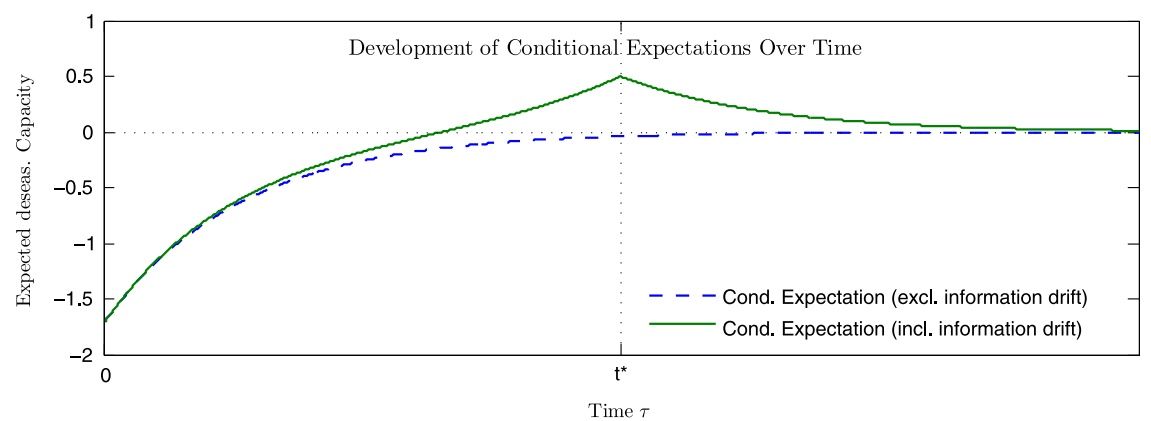

Fig. 3. Development of conditional expectations over time. This figure illustrates the different trajectories of the conditional expectations over time for the de-seasonalized capacity process $m_{t}, \mathbb{E}^{\mathbb{P}}\left[m_{\tau} \mid \mathcal{F}_{t}\right]$ and $\mathbb{E}^{\mathbb{P}}\left[m_{\tau} \mid \mathcal{G}_{t}\right]$, with historical and enlarged filtrations $\mathcal{F}_{t}$ and $\mathcal{G}_{t}$, respectively.

approx. 40 spot price observations on average, given that there are few days in our data sample where no forward quotes have been recorded. Similarly, the six-week in-sample window applied to the Marex Spectron sample requires our model to be fitted to, on average, 26 one-month ahead, 18 two-months ahead, and 30 spot price observations.

Different sets of implied parameter estimates $\Phi_{W}^{\star}$ are obtained for the Bloomberg and Marex Spectron samples (which are priced separately), as well as depending on whether or not forecasts of demand and/or available capacity are used during the estimation procedure. The consequence of using forward-looking information during the estimation (and subsequent) pricing procedure is illustrated in Fig. 3. Using the de-seasonalized capacity process $m_{t}$ as an example, and recalling Eq. (14), the conditional expectation for $m_{t}$ is required as an input to our model for every day within the delivery period of the respective contract. Assuming, as shown in the graph, that on the pricing day, $m_{t}$ amounts to $-1.73 \mathrm{GW}$, the classic "exponential decay" of OU-based processes drives the trajectory of the $\mathcal{F}$-adapted conditional expectation quickly back to the long-run zero-mean. By contrast, if a capacity forecast for, e.g., time $t^{\star}$ is available, the trajectory of $\mathbb{E}^{\mathbb{P}}\left[m_{\tau} \mid \mathcal{G}_{t}\right]$ does not immediately mean-revert to zero, yet instead moves to the forecast point expected to prevail at $\tau=t^{\star}, \mathbb{E}^{\mathbb{P}}\left[m_{t^{\star}} \mid \mathcal{G}_{t}\right]=0.5 \mathrm{GW}$. Note that for $\tau>t^{\star}$, also the trajectory for $\mathbb{E}^{\mathbb{P}}\left[m_{\tau} \mid \mathcal{G}_{t}\right]$ mean-reverts back to zero, given that for $\tau>t^{\star}$, we have the case that $\mathcal{F}=\mathcal{G}$. As a next step, having generated these trajectories over the delivery period of the contract, a model-based forward price can be generated according to Eq. (16), which will then have to be repeated for every spot and forward price quote recorded during the respective in-sample calibration window. The resulting set of model prices will then be fitted to the observed counterparts by iterating this procedure and optimizing over the set of parameters $\Phi_{W}^{\star} \cdot{ }^{28}$

\footnotetext{
${ }^{28}$ Note that although in the above, we refer to $\mathbb{E}^{\mathbb{P}}\left[m_{\tau} \mid \mathcal{G}_{t}\right]$, i.e. to a $\mathbb{P}$-conditional expectation, our pricing procedure will still rely on the respective $\mathbb{Q}$-conditional counterpart since irrespective of using forecast data, the optimization procedure always entails a risk-adjustment via the parameters $\lambda^{D}$ and $\lambda^{C}$.
} 
Finally, we also apply the above mentioned perfect foresight approach, thus establishing another benchmark against which to compare the performance of our pricing model when using forward looking information. Thereby, the realized values of $D_{t}$ and $C_{t}$ for every day during the delivery period of the respective electricity contract will be treated as known in hindsight and will hence replace the conditional expectations in our pricing formula, Eq. (15).

\subsection{Estimation results}

Regarding the processes for electricity demand and available capacity, estimation results and robust standard errors are presented in Tables 2 and 3. Parameter estimates for the dynamics of natural gas are summarized in Table $4 .^{29}$

For the fundamental parameters $\alpha, \beta, \gamma$, and $\delta$, Table 5 summarizes implied estimates based on the Bloomberg sample, when using both demand and capacity forecasts. ${ }^{30}$ Although the table only provides an aggregate view on the estimates, their corresponding means and standard errors indicate significant weekly variation among the parameters which our model could not capture when holding constant these parameters otherwise.

Examining the development of the parameter estimates over time, we observe that $\beta$ and $\gamma$, the parameters governing the sensitivity of the power price with respect to changes in demand and capacity, respectively, culminate in 2008 and gradually decline thereafter. As is further outlined in the next section, this can be well explained by the fact that in terms of excess capacity, the British power market was especially tight in 2008, as is clearly reflected in the behavior of day-ahead and month-ahead forward prices displayed in Fig. 4. The following years are marked by a massive increase in installed generation capacity by more than 10 gigawatts (GW), leading to oversupply especially of thermal generation and, consequently, to tightening spreads (especially spark spreads) for generators. As a consequence of these abundant capacity levels, changes in demand and capacity are of less importance for power price dynamics at that time, as evidenced by rather small absolute values for the estimates of $\beta$ and $\gamma$ in the years 2009-2011. ${ }^{31}$ As will be seen, this strongly affects the relative advantage of using forecasts of demand and capacity.

Recalling that $\delta$ can be interpreted as the elasticity of the power price with respect to changes in the fuel price, we observe that between 2009 and 2011, the estimate for $\delta$ more than doubles. This increase in the power-gas sensitivity may come as a surprise given that at the same time, spark spreads have continued to decline. However, it is the heavily gas-based structure of the British generation park that causes especially the short end of the power price curve to track the NBP gas curve very closely. Hence, the link between gas and power markets may have become even stronger recently, owing to the fact that (i) the UK Large Combustion Plant Directive (LCPD) has started to reduce availability levels of coal plants and that (ii) new generation coming online has primarily been of CCGT-type. ${ }^{32}$ We also note that the increase in value for $\delta$ during 2009-2011 goes in line with a corresponding decrease in value for $\alpha$, which appears reasonable when recalling the interpretation of $\alpha=f_{t}^{1-\delta}$ in Eq. (13).

Finally, in view of rather large estimates for the market prices of demand and capacity risk, $\lambda^{D}$ and $\lambda^{C}$, it is important to mention that since these two parameters are estimated simultaneously, they interact with each other during the estimation procedure and cannot be determined uniquely. It might hence be more convenient to think of a "combined" market price of (reserve) margin risk $\beta \lambda^{D}+\gamma \lambda^{C}$ which is also shown in Table 5.

\section{Pricing results}

In order to examine the pricing impact of using forward-looking information in more detail, we distinguish between four cases: using (i) no forecasts, (ii) demand forecasts only, (iii) forecasts of both demand and available capacity, and (iv) applying the perfect foresight approach described in the previous section. Results are reported for each of the five years covered by our study as well as on an aggregate basis for 2007-2011.

\subsection{Bloomberg sample}

Table 6 summarizes pricing results for 1-month ahead forward contracts from the Bloomberg data set. As can be seen, employing demand and capacity forecasts clearly improves pricing performance on an overall basis (e.g., aggregate RMSPE reduces to less than $6 \%$ as compared to approx. $10 \%$ when no forecasts are used). This result, however, is primarily driven by the strong reductions in pricing errors that can be achieved for the year 2008. As will be outlined below, it is thus mainly during times when electricity prices are particularly sensitive to demand or available capacity that forward-looking

\footnotetext{
${ }^{29}$ In order to preserve space, we do not report the coefficients related to seasonality which are available from the authors upon request. These coefficients are statistically highly significant and underline the distinct seasonalities that are present in the dynamics of both demand, capacity and natural gas.

${ }^{30}$ Estimation results for the other sets of parameters are available from the authors upon request.

31 However, the variation of $\beta$ and $\gamma$ and especially the increase in absolute values for 2008 could, at least to some extent, also be due to insufficient convexity of our functional representation of the merit order curve. The curve is likely to be steeper during times of low system margin than the corresponding levels implied by our exponential-form representation.

${ }^{32}$ Combined cycle gas turbine (CCGT) plants are natural gas fired generation plants which, due to their enhanced technology, achieve high levels of thermal efficiency and offer sufficient flexibility in generation to meet sudden fluctuations in electricity demand.
} 
Table 2

Maximum-likelihood parameter estimates for electricity demand. This table reports Maximum-Likelihood parameter estimates and robust $t$-statistics (White, 1982; [in brackets]) for the discrete-time equivalent of the electricity demand process as specified in Eqs. (2), (3), and (4). Parameters are estimated using five years of daily electricity demand data (in GW units), and are held constant throughout the following year for pricing purposes. Parameter estimates related to seasonality are not reported, yet available from the authors upon request.

\begin{tabular}{|c|c|c|c|c|c|c|c|c|c|c|}
\hline \multirow{2}{*}{$\frac{\text { Period }}{a^{D}}$} & \multicolumn{2}{|c|}{ 2002-2006 } & \multicolumn{2}{|c|}{ 2003-2007 } & \multicolumn{2}{|c|}{ 2004-2008 } & \multicolumn{2}{|c|}{ 2005-2009 } & \multicolumn{2}{|c|}{ 2006-2010 } \\
\hline & 56.05 & [401.72] & 56.78 & [395.75] & 57.25 & [429.66] & 57.90 & [394.80] & 57.80 & [388.69] \\
\hline$b^{D}$ & 0.36 & {$[13.01]$} & 0.01 & {$[0.47]$} & -0.27 & {$[-10.38]$} & -0.69 & {$[-23.81]$} & -0.67 & {$[-23.28]$} \\
\hline$\kappa^{D}$ & 148.20 & [21.47] & 137.92 & [24.03] & 141.93 & [23.49] & 131.14 & [26.53] & 125.12 & [26.69] \\
\hline$\sigma^{D}$ & 23.72 & [40.55] & 23.22 & [39.64] & 22.48 & [40.85] & 22.37 & [41.89] & 22.03 & [41.90] \\
\hline$\theta^{D}$ & 0.41 & [10.43] & 0.40 & {$[9.82]$} & 0.41 & [10.25] & 0.41 & [10.48] & 0.40 & [10.88] \\
\hline$\zeta^{D}$ & 0.21 & [17.63] & 0.21 & [16.33] & 0.21 & [18.11] & 0.22 & [19.42] & 0.23 & [18.33] \\
\hline LogLik & 2984.32 & & 2947.37 & & 2890.18 & & 2879.65 & & 2851.13 & \\
\hline$R_{\text {McFadden }}^{2}$ & 0.30 & & 0.31 & & 0.29 & & 0.31 & & 0.33 & \\
\hline
\end{tabular}

Table 3

Maximum-likelihood parameter estimates for available system capacity. This table reports Maximum-Likelihood parameter estimates and robust $t$ statistics (White, 1982; [in brackets]) for the discrete-time equivalent of the capacity process as specified in Eqs. (6) and (7). Parameters are estimated using five years of data for daily levels of available capacity (in GW units), and are held constant throughout the following year for pricing purposes. Parameter estimates related to seasonality are not reported, yet available from the authors upon request.

\begin{tabular}{|c|c|c|c|c|c|c|c|c|c|c|}
\hline \multirow{2}{*}{$\frac{\text { Period }}{a^{C}}$} & \multicolumn{2}{|c|}{ 2002-2006 } & \multicolumn{2}{|c|}{ 2003-2007 } & \multicolumn{2}{|c|}{ 2004-2008 } & \multicolumn{2}{|c|}{ 2005-2009 } & \multicolumn{2}{|c|}{ 2006-2010 } \\
\hline & 64.30 & [245.80] & 63.96 & [243.35] & 64.78 & [342.91] & 64.09 & [388.07] & 63.32 & [391.66] \\
\hline$b^{C}$ & -0.07 & {$[-1.28]$} & 0.04 & {$[0.56]$} & -0.28 & {$[-6.00]$} & -0.37 & {$[-10.60]$} & -0.07 & {$[-2.14]$} \\
\hline$\kappa^{C}$ & 137.82 & [31.50] & 157.42 & [21.41] & 157.42 & [20.93] & 148.00 & [22.62] & 138.23 & [24.98] \\
\hline$\sigma^{C}$ & 27.37 & [55.87] & 29.26 & [32.27] & 29.24 & [31.05] & 29.94 & [31.64] & 30.88 & [32.86] \\
\hline LogLik & \multicolumn{2}{|l|}{3245.46} & \multicolumn{2}{|l|}{3369.11} & \multicolumn{2}{|l|}{3370.00} & \multicolumn{2}{|l|}{3411.43} & \multicolumn{2}{|l|}{3467.47} \\
\hline$R_{\text {McFadden }}^{2}$ & \multicolumn{2}{|l|}{0.31} & \multicolumn{2}{|l|}{0.30} & \multicolumn{2}{|l|}{0.30} & \multicolumn{2}{|l|}{0.34} & \multicolumn{2}{|l|}{0.38} \\
\hline
\end{tabular}

Table 4

Kalman filter parameter estimates for natural gas. This table reports parameter estimates and robust $t$-statistics (White, 1982; [in brackets]) for the natural gas price process as specified in Eqs. (9) and (10), using the Kalman filter and maximum likelihood estimation. Parameters are estimated based on five years of daily natural gas futures price data (using 1-, 2-, 3-, and 4-months ahead contracts), and are held constant throughout the following year for pricing purposes. Parameter estimates related to seasonality are not reported, yet available from the authors upon request.

\begin{tabular}{|c|c|c|c|c|c|c|c|c|c|c|}
\hline \multirow{2}{*}{$\begin{array}{l}\text { Period } \\
a^{g}\end{array}$} & \multicolumn{2}{|c|}{ 2002-2006 } & \multicolumn{2}{|c|}{ 2003-2007 } & \multicolumn{2}{|c|}{ 2004-2008 } & \multicolumn{2}{|c|}{ 2005-2009 } & \multicolumn{2}{|c|}{ 2006-2010 } \\
\hline & 0.11 & [7.70] & -0.09 & {$[-2.77]$} & -0.11 & {$[-3.88]$} & 0.09 & [4.57] & -0.16 & [-8.88] \\
\hline$b^{g}$ & 0.56 & [9.52] & 1.04 & [9.07] & -0.08 & {$[-1.26]$} & -0.08 & {$[-2.91]$} & -0.31 & {$[-6.22]$} \\
\hline$\kappa^{g}$ & 0.26 & [5.13] & 0.06 & [5.87] & 0.13 & [6.45] & 0.29 & [10.68] & 0.23 & [10.80] \\
\hline$\lambda^{g}$ & 3.72 & [19.75] & 7.54 & [7.70] & 7.74 & [12.46] & 5.63 & [28.02] & 6.38 & [24.20] \\
\hline$\sigma^{g}$ & 0.47 & [13.28] & 0.49 & [15.67] & 0.49 & [15.55] & 0.55 & [15.34] & 0.53 & [16.51] \\
\hline LogLik & \multicolumn{2}{|c|}{11067.23} & \multicolumn{2}{|c|}{10535.82} & \multicolumn{2}{|c|}{10357.80} & \multicolumn{2}{|c|}{10749.18} & \multicolumn{2}{|l|}{10972.14} \\
\hline$R_{\text {McFadden }}^{2}$ & \multicolumn{2}{|l|}{0.23} & \multicolumn{2}{|l|}{0.24} & \multicolumn{2}{|l|}{0.28} & \multicolumn{2}{|l|}{0.30} & \multicolumn{2}{|l|}{0.26} \\
\hline
\end{tabular}

information can help to improve the pricing performance of our model significantly. However, even if the reduction of pricing errors is much smaller, e.g., during 2009 or 2011, such improvements in pricing performance, nevertheless, are still of economic significance to market participants - especially in view of average contract volumes of several thousands of megawatt hours (MW h).

In order for the analysis of pricing errors to be consistent with our estimation procedure, we mainly focus on root mean squared-based error measures, given that this objective function has also been used for estimation. However, we also note that the relative improvement in pricing performance when employing forecasts is generally smaller when looking at the absolute percentage error (MAPE) as opposed to RMSPE, which underlines our general conclusion that incorporating forecasts seems to pay off mainly in situations of unusually high demand or low capacity. Hence, before analyzing the breakdown of pricing errors on a yearly basis, it is important to recall that primarily during the first 2-2.5 years of our study, the British power market has suffered from exceptionally poor expected levels of power plant availability, with reserve margins clearly falling below long-term averages, especially in 2008. Consequently, the model excluding forecasts fares clearly worse than during any other period of our study. By contrast, the model including both demand and capacity forecasts gives strong evidence of its capabilities, reducing pricing errors even in times of extreme fluctuations in day-ahead 
Table 5

Implied parameter estimates for $\Phi_{W}^{\star}$ (Bloomberg sample/forecasts for $D_{t}$ and $C_{t}$ ). This table reports yearly average values and corresponding standard errors [in brackets] of the implied estimates for the "fundamental" and risk-neutral parameters $\Phi_{W}^{\star}=\left\{\alpha, \beta, \gamma, \delta, \lambda_{\mathcal{G}}^{D}, \lambda_{\mathcal{G}}^{C}\right\}$. Parameters are obtained by minimizing the root mean squared percentage errors (RMSPE) between observed market prices and theoretical model prices for both 1-day ahead and 1-month ahead forward contracts from the Bloomberg (BBG) data sample. Throughout the estimation procedure, forecasts of both electricity demand and available capacity are used. In-sample estimation is performed based on a time window $W$ of eight weeks with weekly shifting. Then, for every parameter in $\Phi_{W}^{\star}$, the below displayed average values are computed based on the set of estimates implied from all in-sample windows in the respective year.

\begin{tabular}{|c|c|c|c|c|c|c|}
\hline Parameter & 2007 & 2008 & 2009 & 2010 & 2011 & 2007-2011 \\
\hline \multirow[t]{2}{*}{$\alpha$} & 1.5203 & 3.0155 & 2.7241 & 2.0338 & 1.8217 & 2.2220 \\
\hline & {$[0.1386]$} & {$[0.1956]$} & {$[0.0981]$} & {$[0.1025]$} & {$[0.1276]$} & [0.0697] \\
\hline \multirow[t]{2}{*}{$\beta$} & 0.0279 & 0.0286 & 0.0164 & 0.0135 & 0.0106 & 0.0193 \\
\hline & {$[0.0020]$} & [0.0027] & [0.0009] & [0.0008] & [0.0003] & {$[0.0008]$} \\
\hline \multirow[t]{2}{*}{$\gamma$} & -0.0175 & -0.0344 & -0.0150 & -0.0116 & -0.0117 & -0.0180 \\
\hline & [0.0015] & {$[0.0035]$} & [0.0006] & {$[0.0004]$} & {$[0.0004]$} & [0.0009] \\
\hline \multirow[t]{2}{*}{$\delta$} & 0.4317 & 0.4369 & 0.2713 & 0.4674 & 0.5578 & 0.4324 \\
\hline & [0.0334] & [0.0388] & [0.0269] & [0.0253] & [0.0317] & {$[0.0151]$} \\
\hline \multirow[t]{2}{*}{$\lambda_{\mathcal{G}}^{D}$} & -16.3475 & 3.9719 & -45.1915 & -30.9245 & -58.2689 & -29.5412 \\
\hline & [10.2246] & {$[14.4244]$} & [12.5963] & [7.5293] & [8.8900] & [5.0647] \\
\hline \multirow{2}{*}{$\lambda_{\mathcal{G}}^{C}$} & 20.2954 & 11.3382 & 52.4444 & 36.0655 & 64.8668 & 37.1602 \\
\hline & [15.0584] & [18.2663] & [14.5850] & [7.6381] & [9.5236] & [6.1353] \\
\hline \multirow[t]{2}{*}{$\beta \lambda_{\mathcal{G}}^{D}+\gamma \lambda_{\mathcal{G}}^{C}$} & -0.0817 & -0.8503 & -1.5816 & -0.6957 & -1.2935 & -0.9034 \\
\hline & {$[0.4304]$} & [0.8125] & {$[0.4360]$} & [0.1589] & [0.1913] & [0.2087] \\
\hline
\end{tabular}
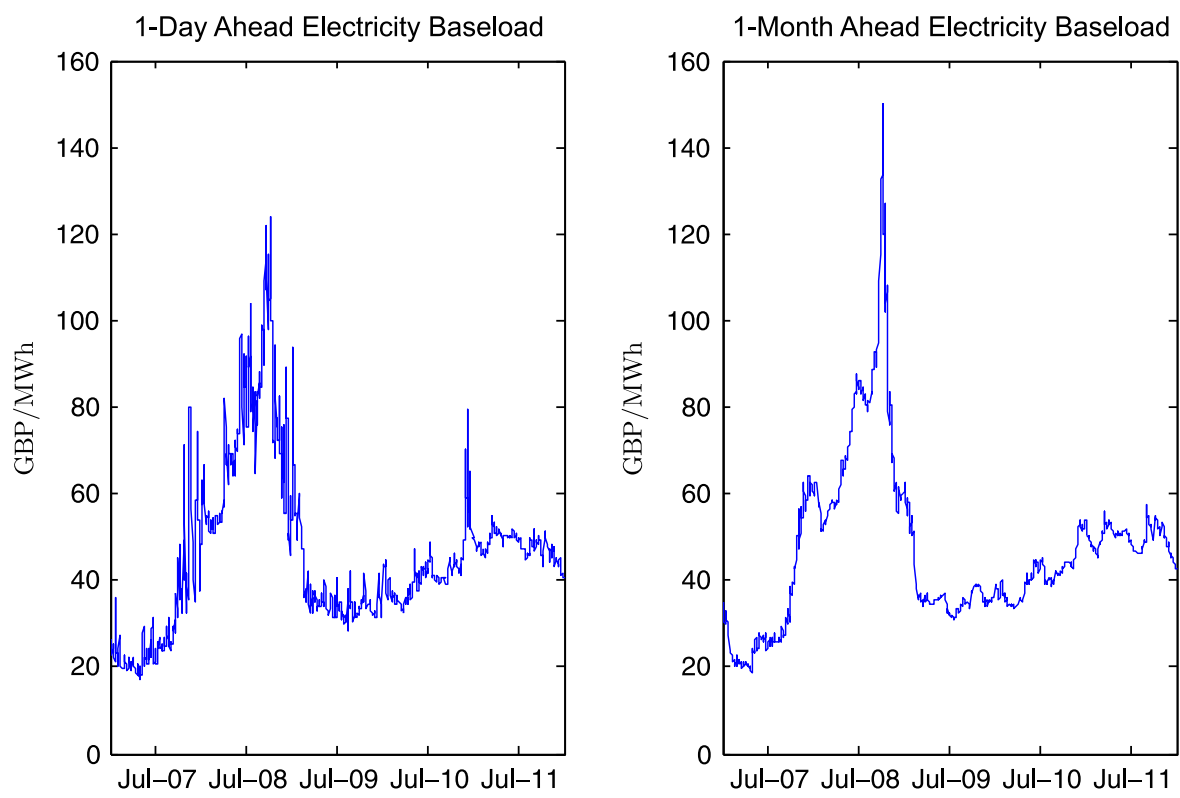

Fig. 4. 1-Month ahead forward and 1-day ahead baseload electricity prices. This figure shows the time series of daily forward prices for 1-month ahead and 1-day ahead baseload electricity contracts during the period from 1 January 2007 to 31 December 2011. All data shown were obtained from Bloomberg; for dates with missing quotes/prices, the last observed historic price was carried over.

and forward price levels, i.e., during times demanding utmost flexibility from any type of model. Reconsidering Fig. 4, the extreme spike in month-ahead forward prices during September/October 2008 was clearly driven by ever-increasing supply fears, ${ }^{33}$ and it is obvious that such a trajectory can only be captured (albeit not perfectly) by a model that includes forward-looking information about the capacity levels that are expected to prevail during the respective delivery months. ${ }^{34}$

\footnotetext{
${ }^{33}$ This is supported by our analysis of market commentary covering the respective trading days. Importantly, in these days, prices of month-ahead natural gas were approximately flat.

34 The benefit of using forecasts during times of high demand and/or tight reserve margins can also be confirmed by regressing the related reduction in pricing errors on a measure that quantifies by how much the forecasts deviate from corresponding long-term seasonal levels. More precisely, on every pricing day, we feed into our model estimates of both demand and available capacity that are expected to prevail on every day within the delivery period of the respective contract (see Eq. (16)). Thus, for every delivery day, we compute the percentage deviation between these sets of demand (capacity) expectations when based on forward-looking information and when excluding it. For our regression, we define the regressors as the maximum of these demand (capacity) deviations, i.e., where on every pricing day, the maximum is taken over all delivery days. Especially for the years 2007 and 2008 , regressing the reduction in RMSPE on these regressors yields highly statistically significant coefficients at the $1 \%$-level.
} 
Table 6

Out-of-sample pricing results: 1-month ahead forward contracts (BBG). This table reports yearly (and aggregate) pricing errors for 1-month ahead electricity forward contracts from the Bloomberg (BBG) data sample; implemented models either do not rely on forecasts ("No FC") or incorporate demand and/or capacity forecasts ("FC for $D_{t}$ " and "FC for $D_{t}$ and $C_{t}$ ", respectively). In addition, results for the perfect-foresight case are reported. We use weekly subperiods for out-of-sample pricing, with in-sample fitting of the model being performed based on the cross-section of 1-day ahead and 1-month ahead forward quotes collected during the preceding eight weeks. Error measures shown are mean percentage error (MPE), mean absolute percentage error (MAPE), root mean squared error (RMSE), and root mean squared percentage error (RMSPE).

\begin{tabular}{|c|c|c|c|c|c|c|c|c|}
\hline Model & MPE & MAPE & RMSE & RMSPE & MPE & MAPE & RMSE & RMSPE \\
\hline & \multicolumn{4}{|l|}{2007} & \multicolumn{4}{|l|}{2008} \\
\hline No FC & $-3.33 \%$ & $5.19 \%$ & $£ 2.90$ & $7.17 \%$ & $0.98 \%$ & $9.83 \%$ & $£ 17.22$ & $20.57 \%$ \\
\hline FC for $D_{t}$ & $-4.54 \%$ & $5.86 \%$ & $£ 3.78$ & $7.76 \%$ & $0.71 \%$ & $7.73 \%$ & $£ 10.29$ & $12.04 \%$ \\
\hline $\mathrm{FC}$ for $D_{t}$ and $C_{t}$ & $-4.30 \%$ & $5.53 \%$ & $£ 3.47$ & $7.49 \%$ & $-1.19 \%$ & $6.65 \%$ & $£ 7.73$ & $8.99 \%$ \\
\hline \multirow[t]{2}{*}{ Perfect foresight } & $-3.90 \%$ & $5.13 \%$ & $£ 3.10$ & $6.66 \%$ & $0.24 \%$ & $7.67 \%$ & $£ 11.04$ & $12.84 \%$ \\
\hline & \multicolumn{4}{|l|}{2009} & \multicolumn{4}{|l|}{2010} \\
\hline No FC & $-0.79 \%$ & $3.18 \%$ & $£ 2.06$ & $5.99 \%$ & $-1.02 \%$ & $2.00 \%$ & $£ 1.31$ & $2.96 \%$ \\
\hline FC for $D_{t}$ & $-0.11 \%$ & $2.68 \%$ & $£ 1.36$ & $3.90 \%$ & $-0.36 \%$ & $1.94 \%$ & $£ 1.16$ & $2.69 \%$ \\
\hline $\mathrm{FC}$ for $D_{t}$ and $C_{t}$ & $-0.28 \%$ & $2.83 \%$ & $£ 1.46$ & $4.16 \%$ & $-0.31 \%$ & $2.17 \%$ & $£ 1.32$ & $3.03 \%$ \\
\hline \multirow[t]{2}{*}{ Perfect foresight } & $-0.25 \%$ & $2.74 \%$ & $£ 1.43$ & $4.09 \%$ & $-0.44 \%$ & $1.83 \%$ & $£ 1.16$ & $2.65 \%$ \\
\hline & \multicolumn{4}{|l|}{2011} & \multicolumn{4}{|c|}{ 2007-2011 } \\
\hline No FC & $1.15 \%$ & $2.59 \%$ & $£ 1.73$ & $3.63 \%$ & $-0.62 \%$ & $4.56 \%$ & $£ 7.90$ & $10.31 \%$ \\
\hline FC for $D_{t}$ & $1.06 \%$ & $2.18 \%$ & $£ 1.37$ & $2.81 \%$ & $-0.67 \%$ & $4.09 \%$ & $£ 5.00$ & $6.87 \%$ \\
\hline FC for $D_{t}$ and $C_{t}$ & $1.47 \%$ & $2.41 \%$ & $£ 1.57$ & $3.23 \%$ & $-0.94 \%$ & $3.93 \%$ & $£ 3.95$ & $5.91 \%$ \\
\hline Perfect foresight & $1.33 \%$ & $2.29 \%$ & $£ 1.46$ & $3.02 \%$ & $-0.62 \%$ & $3.94 \%$ & $£ 5.23$ & $6.96 \%$ \\
\hline
\end{tabular}

Regarding the perfect foresight approach, it is interesting to note that pricing errors generally tend to be lower than for any other of the approaches implemented - however, except for the year 2008. Here, the RMSPE under perfect foresight amounts to $12.84 \%$, almost $4 \%$ above the model variant that fully uses demand and capacity forecasts. Interestingly, one may argue that the forecasts seem to have had higher informational content than the ex-post realized values as regards price discovery in the forward market. Consequently, the market - at least for this year - seems to have "traded on" these forecast values rather than on realized data; for the other years, as we would have expected, the perfect foresight approach yields the best results. However, as mentioned above, electricity forward prices generally seem to be less sensitive to the underlying factors demand and capacity during the years 2010 and 2011, which slightly limits the potential for further analyses in this regard.

The pricing performance of the models during the year 2007 provides another opportunity to further discuss what kind of forward-looking information we actually consider to be contained in the enlarged filtration $\left(\mathcal{G}_{t}^{C}\right)_{t \in\left[0, T^{\star}\right]}$ - and what is not contained therein. Based on a detailed analysis of single-day pricing errors, the model including both forecast types yields very satisfactory pricing results throughout this year, except for a period of rather poor pricing performance during November and December 2007, for which forward prices are clearly underestimated. Although market commentary may generally be criticized for over-emphasizing alleged causal relationships between specific events and strong market movements, several reports released at that time stress, amongst other reasons, the then very high continental power prices that are said to have impacted British power prices as well. In fact, French power prices had reached record levels in November 2007, fueled by strikes in the energy sector that led to temporary production cuts of about 8000 MW. This, in turn, raised concerns about French electricity supplies for the rest of the year, which ultimately could have resulted in Britain becoming a net exporter of power to France via its interconnector, putting an additional drain on the already tight British system. ${ }^{35}$ However, although market commentary indicates that (British) market participants do seem to have "priced in" such a scenario, and although pricing errors for the forecast-based variant of our model would have clearly been reduced, we have decided not to incorporate this belief (i.e., a longer-lasting strike in France having interconnectors switch from imports to exports) into our capacity forecasts: $\left(\mathcal{G}_{t}^{C}\right)_{t \in\left[0, T^{\star}\right]}$ is only based on forecasts released by the TSO and supplemented with updates of major unplanned outages. Although likely to further improve pricing performance, starting to integrate market beliefs about future available import/export capacity levels would also require us to do so for the rest of our sample, i.e., during times where such market sentiment may be more difficult to infer. Moreover, it is obviously impossible to exactly observe and consistently quantify these beliefs. For instance, it is unknown how long exactly and to what extent market participants would expect the above scenario of strikes in the French energy sector to continue.

\footnotetext{
${ }^{35}$ The interconnector that links British and French electricity markets has a capacity of approx. 2000 MW; Britain has "traditionally" been an importer of French electricity - which especially during peak hours tends to be cheaper, also in view of the higher share of nuclear baseload generation capacity. Yet at that time in 2007, it was feared that the strike would cause electricity in France to become more expensive than in Britain, thus reverting the usual direction of interconnector flows.
} 

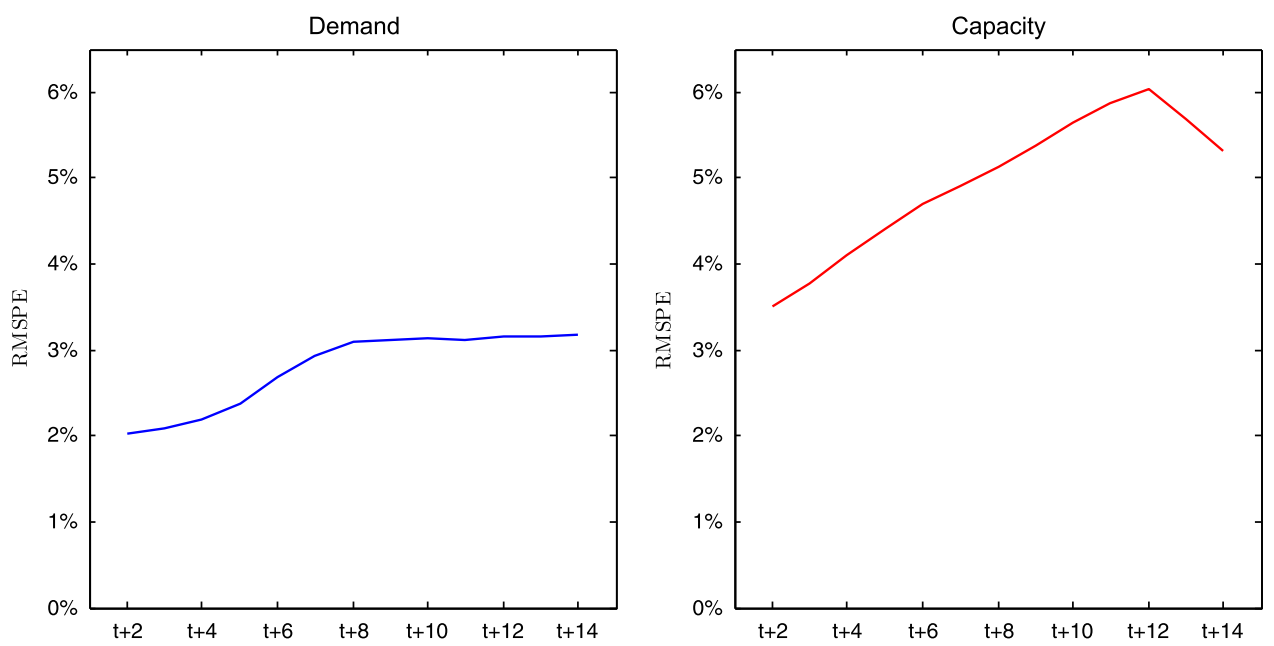

Fig. 5. Performance of demand and capacity forecasts. This figure shows the root mean squared percentage error (RMSPE) for the 2-14 days-ahead forecasts of electricity demand and available system capacity during the period from 1 January 2007 to 31 December 2011. Note that especially for capacity forecasts, inputs are based on data released by National Grid plc, after adjustments by the authors.

In the years 2009-2011, the relative improvement of the forecast-based models is smaller than in previous years. As indicated by the corresponding parameter estimates for $\beta$ and $\gamma$, the influence of demand and capacity as fundamental factors driving power prices has been much reduced during these years, primarily due to growing oversupply in generation capacities leading to permanently higher reserve margin levels. Given that short- to mid-term power prices at that time were almost exclusively driven by natural gas dynamics under these conditions, the impact of incorporating forwardlooking information vanishes accordingly. Interestingly, pricing performance of the model for the years 2009-2011 seems to be even slightly better when using demand forecasts only, and leaving capacity forecasts aside. As also stressed by Karakatsani and Bunn (2008), this could be due to the fact that in the British market, the forecasts of available capacity levels (or, equivalently, margins) released by the TSO tend to be received with slight skepticism and, hence, are likely to be adjusted (or not used at all) by market players.

This leads to other, more general problems of capacity forecasts, such as their accuracy in terms of generation from renewables or their consistency in definition with realized data. This is also reflected in Fig. 5 where prediction errors between forecast and realized demand and capacity levels are summarized, using the RMSPE as the relevant error metric. ${ }^{36}$ Capturing well the regular consumption patterns that characterize the dynamics of electricity demand, related forecasts are subject to rather low forecast errors only: for a forecast horizon of up to the next 8 days, the RMSPE increases steadily from $2 \%$ for short-term forecasts to approx. $3 \%$ for forecasts of electricity demand expected one week ahead. For longer forecast horizons, the forecast error increases at a visibly slower rate; although not within the scope of this paper, one may argue that this "break" could be due to the underlying (weather) forecast model transitioning from daily (point-) forecasts to a lower-resolution model that will be likely to rely more strongly on statistical averages than on the weather/demand situation prevailing on day $t=0 .{ }^{37}$ By contrast, predicted future levels of available capacity are significantly less accurate and this inaccuracy increases more strongly for longer forecast horizons. In fact, for certain time periods within our data sample, expected capacity levels would even have been predicted more accurately when leaving out forward-looking information altogether. Also, in contrast to what can be observed for electricity demand forecasts, Fig. 5 does not show a similar influence of statistical averages that would prevent the RMSPE from increasing for longer time periods. While this certainly impacts pricing performance during 2009-2011, i.e. during times of high sensitivity of electricity prices to underlying factors of demand and capacity, such generally higher inaccuracy of capacity forecasts nevertheless seems to be of minor importance during times of exceptionally low reserve margins, as shown above.

\footnotetext{
${ }^{36}$ Note that especially for forecasts of available capacity, the input capacity data from National Grid has been subject to further adjustments by the authors. The impact on the pricing performance of our model is negligible for the outer years covered by our study (change in RMSPE below $0.5-1.0 \%$ for the Bloomberg and Marex Spectron datasets, respectively, when forecasts for both $D_{t}$ and $C_{t}$ are used); for the years 2007 and 2008 , the impact is clearly stronger (RMSPE increases by approx. 3.0-5.0\%) given a generally slightly higher number of missing data points especially in 2007 and, as mentioned, due to comparatively more unexpected outages occurring during 2008.

${ }^{37}$ In fact, it can be shown that the development of the empirical variance of $\mathbb{E}^{\mathbb{P}}\left[D_{\tau} \mid \mathcal{G}_{t}\right]$ for different forecast horizons $\tau$ follows a very similar pattern as in Fig. 5. Thereby, a "kink" at around $\tau=8$ can again be perceived, and the influence of statistical averages driving weather forecasts for longer forecast horizons again becomes obvious, given that the variance of $\mathbb{E}^{\mathbb{P}}\left[D_{\tau} \mid \mathcal{G}_{t}\right]$ for $\tau>8$ strongly converges to the historical variance implied from our parameter estimates in Table 2. For further information on weather forecasting models and their underlying structures, also see, e.g., Lynch et al. (2014) and the literature cited therein.
} 


\subsection{Marex Spectron sample}

The results based on the data obtained from Marex Spectron are presented in Tables 7 and 8. Again, we observe an improvement in pricing performance when integrating demand and capacity forecasts into our model, as evidenced by relative reductions in total RMSPE of $8 \%$ and $15 \%$ for 1-month and 2-months ahead forward contracts, respectively. Moreover, the overall pattern of pricing errors for both types of forward contracts is in line with the conclusions drawn from the Bloomberg sample. Notably, integrating demand as well as capacity forecasts into our model again primarily pays off during the years 2007-2008, reducing aggregate RMSE during these years by about $£ 1.20-2.00 / M W$ h. Such economic significance is also confirmed statistically by applying a Wilcoxon signed-rank test which shows that the reductions in errors

Table 7

Out-of-sample pricing results: 1-month ahead forward contracts (OTC). This table reports yearly (and aggregate) pricing errors for 1-month ahead electricity forward contracts from the Marex Spectron (OTC) data sample; implemented models either do not rely on forecasts ("No FC") or incorporate demand and/or capacity forecasts ("FC for $D_{t}$ " and "FC for $D_{t}$ and $C_{t}$ ", respectively). In addition, results for the perfect-foresight case are reported. We use weekly subperiods for out-of-sample pricing, with in-sample fitting of the model being performed based on the cross-section of 1-day ahead, 1-month ahead, and 2-months ahead forward quotes collected during the preceding six weeks. Error measures shown are mean percentage error (MPE), mean absolute percentage error (MAPE), root mean squared error (RMSE), and root mean squared percentage error (RMSPE).

\begin{tabular}{|c|c|c|c|c|c|c|c|c|}
\hline Model & MPE & MAPE & RMSE & RMSPE & MPE & MAPE & RMSE & RMSPE \\
\hline & \multicolumn{4}{|l|}{2007} & \multicolumn{4}{|l|}{2008} \\
\hline No FC & $-2.73 \%$ & $6.11 \%$ & $£ 4.50$ & $8.97 \%$ & $-2.78 \%$ & $7.18 \%$ & $£ 9.04$ & $9.51 \%$ \\
\hline $\mathrm{FC}$ for $D_{t}$ & $-3.60 \%$ & $6.14 \%$ & $£ 4.26$ & $8.57 \%$ & $-1.88 \%$ & $8.11 \%$ & $£ 9.25$ & $10.23 \%$ \\
\hline FC for $D_{t}$ and $C_{t}$ & $-3.16 \%$ & $5.77 \%$ & $£ 3.90$ & $7.74 \%$ & $-1.17 \%$ & $6.71 \%$ & $£ 7.49$ & $8.60 \%$ \\
\hline \multirow[t]{2}{*}{ Perfect foresight } & $-3.02 \%$ & $5.72 \%$ & $£ 3.94$ & $7.80 \%$ & $-1.68 \%$ & $7.14 \%$ & $£ 8.08$ & $9.01 \%$ \\
\hline & \multicolumn{4}{|l|}{2009} & \multicolumn{4}{|l|}{2010} \\
\hline No FC & $2.44 \%$ & $3.34 \%$ & $£ 1.78$ & $4.65 \%$ & $0.13 \%$ & $2.32 \%$ & $£ 1.38$ & $3.03 \%$ \\
\hline $\mathrm{FC}$ for $D_{t}$ & $2.07 \%$ & $3.15 \%$ & $£ 1.63$ & $4.31 \%$ & $0.12 \%$ & $2.25 \%$ & $£ 1.37$ & $2.98 \%$ \\
\hline $\mathrm{FC}$ for $D_{t}$ and $C_{t}$ & $2.52 \%$ & $3.38 \%$ & $£ 1.76$ & $4.63 \%$ & $0.33 \%$ & $2.40 \%$ & $£ 1.44$ & $3.21 \%$ \\
\hline \multirow[t]{2}{*}{ Perfect foresight } & $2.50 \%$ & $3.28 \%$ & $£ 1.72$ & $4.52 \%$ & $0.34 \%$ & $2.24 \%$ & $£ 1.35$ & $2.98 \%$ \\
\hline & \multicolumn{4}{|l|}{2011} & \multicolumn{4}{|c|}{ 2007-2011 } \\
\hline No FC & $2.25 \%$ & $3.18 \%$ & $£ 1.87$ & $3.82 \%$ & $-\mathbf{0 . 0 2 \%}$ & $4.30 \%$ & $£ 4.52$ & $6.40 \%$ \\
\hline $\mathrm{FC}$ for $D_{t}$ & $1.81 \%$ & $3.03 \%$ & $£ 1.74$ & $3.61 \%$ & $-0.19 \%$ & $4.39 \%$ & $£ 4.53$ & $6.42 \%$ \\
\hline FC for $D_{t}$ and $C_{t}$ & $2.36 \%$ & $3.54 \%$ & $£ 2.05$ & $4.19 \%$ & $0.28 \%$ & $4.26 \%$ & $£ 3.87$ & $5.91 \%$ \\
\hline Perfect foresight & $2.27 \%$ & $3.26 \%$ & $£ 1.89$ & $3.87 \%$ & 0.19\% & $4.21 \%$ & $£ 4.07$ & $5.95 \%$ \\
\hline
\end{tabular}

Table 8

Out-of-sample pricing results: 2-months ahead forward contracts (OTC). This table reports yearly (and aggregate) pricing errors for 2-months ahead electricity forward contracts from the Marex Spectron (OTC) data sample; implemented models either do not rely on forecasts ("No FC") or incorporate demand and/or capacity forecasts ("FC for $D_{t}$ " and "FC for $D_{t}$ and $C_{t}$ ", respectively). In addition, results for the perfect-foresight case are reported. We use weekly subperiods for out-of-sample pricing, with in-sample fitting of the model being performed based on the cross-section of 1-day ahead, 1-month ahead, and 2-months ahead forward quotes collected during the preceding six weeks. Error measures shown are mean percentage error (MPE), mean absolute percentage error (MAPE), root mean squared error (RMSE), and root mean squared percentage error (RMSPE).

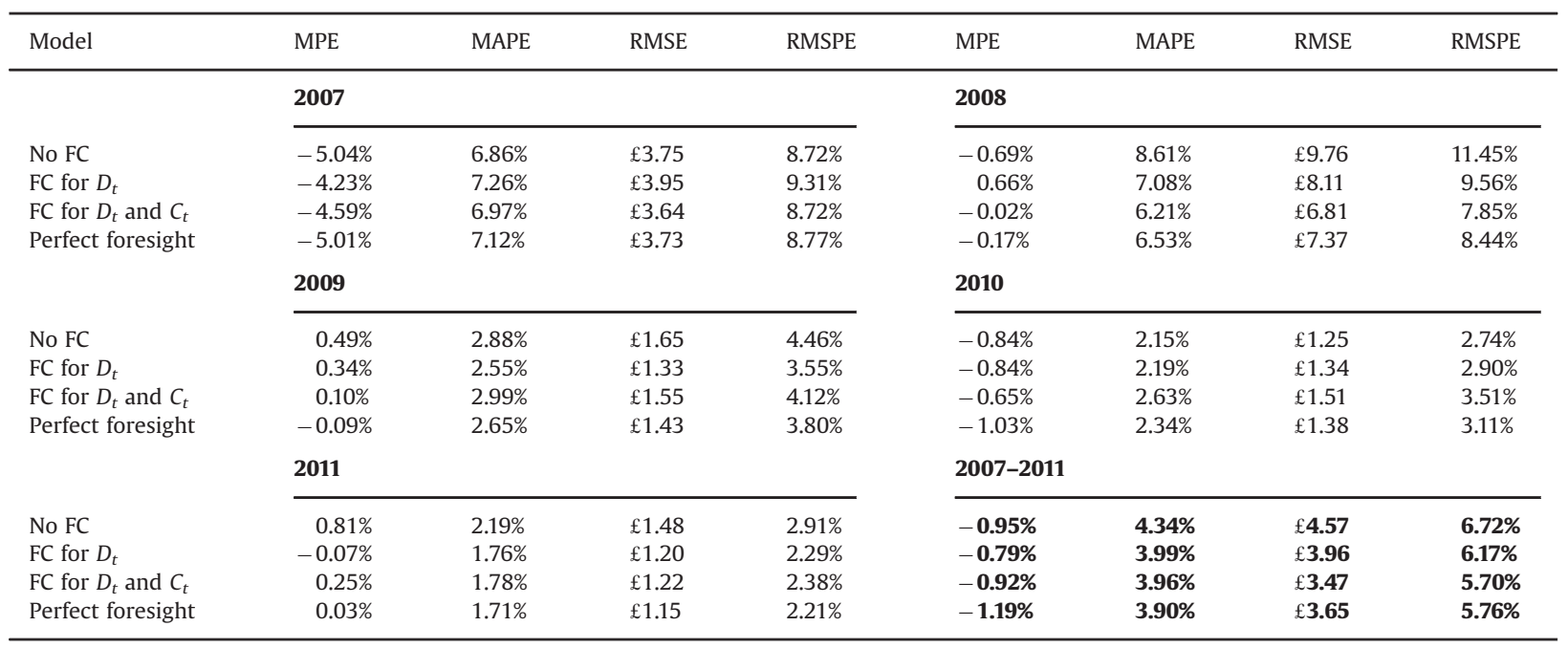


are significant at the 1\%-level. For the remaining years, during which the impact of the fundamental factors $D_{t}$ and $C_{t}$ has been found to be rather muted, pricing errors can still be reduced by using only demand forecasts as compared to the "noforecast" case. The perfect foresight case also shows a pattern in pricing errors that is similar to the Bloomberg sample. In fact, pricing performance in the year 2008 again is worse than if forecasts are used, which is in line with our previous conjecture that forward-looking information in this year may have driven price discovery to a higher extent than ex-post realized values, which appears intuitive given the average maturity of 45 days for 2 -months ahead forward contracts in our sample.

Obviously, the differences in error metrics between the models including and excluding forward-looking information are not of the same order of magnitude as those reductions in pricing errors observed for the Bloomberg sample. Importantly, however, the in-sample fitting procedure for the Marex Spectron data sample additionally includes 2-months ahead forward contracts. As such, when comparing the different pricing results for the Bloomberg sample (Table 6) and the OTC sample (Table 7), our pricing model needs to be fitted to a slightly longer part of the forward curve, i.e., 1-month and 2-months contracts, as compared to only reproducing the short end as in the case of the Bloomberg sample. As mentioned, in order to keep our pricing results still comparable, the estimation window for the OTC case was slightly shortened so that the requirement for the model to also reproduce 2-months ahead contracts is slightly relaxed by reducing the overall number of prices per estimation window. The fact that the pricing results in Tables 6 and 7 are not strongly diverging but rather similar for several years may be seen as confirming such balance induced by a slightly shorter pricing window. In the end, the fact that the benefits of using forecasts still prevail when calibrating our model to a broader cross-section of forward quotes may clearly be seen as underlining the robustness of our general findings. ${ }^{38}$

Examining the pricing errors in more detail, the year 2008 may again serve as an example to illustrate another and more subtle effect when using forecasts as compared to excluding them. For this year, and based on the Bloomberg data sample, pricing performance of the "no-forecast" variant of the model is especially poor, as indicated by an RMSPE of about 20\%. For the Marex Spectron sample, by contrast, corresponding pricing errors for 1-month ahead contracts are much lower, yielding an RMSPE of less than $10 \%$. In this context, it is important to note that amidst the height of above mentioned capacity shortage in 2008 (that led to the prominent spike in 1-month ahead forward prices in September/October shown in Fig. 4), supply fears primarily concentrated on the front month. Consequently, 2-months ahead forward contracts at that time were clearly less subject to such strong fluctuations in price levels. Therefore, the broader cross-section of forward quotes in the Marex Spectron sample forces the "no-forecast" variant of our model to simultaneously accommodate such contrary 1month and 2-months ahead price dynamics, which results in a "mediocre compromise" at best: 1-month ahead contracts are now strongly underestimated (2008 MPE of -2.78\% in Table 7 vs. $0.98 \%$ in Table 6), which, however, halves RMSPE to less than $10 \%$, given that underpricing pays off after the sudden "collapse" in post-spike forward pricing levels. Yet, on the other hand, the pronounced spike in 1-month ahead forwards has 2-months ahead contracts become strongly overpriced postspike (despite an overall 2008 MPE of - 0.69\%), which alone contributes more than $2 \%$ to the overall RMSPE of $11.45 \%$. By contrast, and again comparing Tables 6 and 7, all pricing errors for the model including demand and capacity forecasts in 2008 are surprisingly similar, irrespective of whether or not 2-months ahead contracts are included in the cross-section.

Put differently, the above example provides evidence of the additional benefits that arise when including forecasts into our model. Forecasting low levels of capacity in the short-term, but higher levels in the mid- to long-term may help govern opposed dynamics of contracts with differing maturities, such as outlined above. This flexibility is also reflected in the implicitly estimated fundamental parameters $\alpha, \beta, \gamma$, and $\delta$ as stated in Table 5 and as compared to Table 9. In fact, the implied estimates for $\beta$, and especially for $\gamma$, tend to show higher variation throughout 2007 and 2008 than if demand and/or capacity forecasts are accounted for during the estimation procedure.

This appears reasonable given the additional flexibility for the forecast-based model variants in fitting observed prices, whereas the model variant without forecasts always has expected demand and capacity mean-revert to the same longterm levels. As a result, flexibility is reduced, which must be compensated for both by higher variation within the set of fundamental parameters. Also note that in Table 9, absolute values for $\beta$ and $\gamma$ in the years 2007 and 2008 are slightly higher as compared to the estimates in Table 5. Given that these parameters, ultimately, govern the steepness of the "stylized" merit-order curve within our framework, we can see that expected values of, e.g., available capacity (which in the no-forecast case often are too high) must be compensated for by more negative values for $\gamma$ : without using forwardlooking information, we thus obtain an estimate for $\gamma$ of -0.0522 in the year 2007, as compared to -0.0344 when forecasts are used. Altogether, this again underlines that excluding forecasts from the pricing procedure not only affects pricing performance, but may also imply using a mis-specified model.

\section{Conclusion}

Modeling the dynamics of electricity prices has traditionally been a challenging task for market participants, such as generators/suppliers, traders, and speculators. The strong links between power prices and their fundamental drivers make structural modeling approaches especially appealing in this context, and it can be expected that both current and future

${ }^{38}$ As a further robustness check, the in-sample estimation window was shortened from 6 to 4 weeks, yielding similar pricing results. In order to preserve space, these results are not reported here, yet available from the authors upon request. 
Table 9

Implied parameter estimates for $\Phi_{W}^{\star}$ (Bloomberg sample/no forecasts for $D_{t}$ and $C_{t}$ ). This table reports yearly average values and corresponding standard errors [in brackets] of the implied estimates for the "fundamental" and risk-neutral parameters $\Phi_{W}^{\star}=\left\{\alpha, \beta, \gamma, \delta, \lambda_{\mathcal{F}}^{D}, \lambda_{\mathcal{F}}^{C}\right\}$. Parameters are obtained by minimizing the root mean squared percentage errors (RMSPE) between observed market prices and theoretical model prices for both 1-day ahead and 1month ahead forward contracts from the Bloomberg (BBG) data sample. Throughout the estimation procedure, no forecasts - neither of electricity demand nor of available capacity are used. In-sample estimation is performed based on a time window $W$ of eight weeks with weekly shifting. Then, for every parameter in $\Phi_{W}^{\star}$, the below displayed average values are computed based on the set of estimates implied from all in-sample windows in the respective year.

\begin{tabular}{|c|c|c|c|c|c|c|}
\hline Parameter & 2007 & 2008 & 2009 & 2010 & 2011 & 2007-2011 \\
\hline \multirow[t]{2}{*}{$\alpha$} & 2.1137 & 3.5785 & 2.8118 & 2.4710 & 2.1326 & 2.6186 \\
\hline & [0.1739] & [0.1547] & [0.1077] & {$[0.0804]$} & [0.1196] & {$[0.0672]$} \\
\hline \multirow[t]{2}{*}{$\beta$} & 0.0460 & 0.0363 & 0.0227 & 0.0174 & 0.0106 & 0.0266 \\
\hline & [0.0048] & {$[0.0034]$} & [0.0011] & [0.0015] & {$[0.0002]$} & [0.0015] \\
\hline \multirow[t]{2}{*}{$\gamma$} & -0.0482 & -0.0500 & -0.0265 & -0.0218 & -0.0154 & -0.0323 \\
\hline & {$[0.0064]$} & {$[0.0038]$} & {$[0.0018]$} & {$[0.0021]$} & [0.0009] & {$[0.0018]$} \\
\hline \multirow[t]{2}{*}{$\delta$} & 0.4924 & 0.4247 & 0.3367 & 0.4505 & 0.5295 & 0.4464 \\
\hline & [0.0449] & {$[0.0361]$} & {$[0.0285]$} & [0.0303] & [0.0334] & {$[0.0161]$} \\
\hline \multirow[t]{2}{*}{$\lambda_{\mathcal{G}}^{D}$} & -0.1513 & -0.0929 & -2.5398 & -1.0939 & 0.7940 & -0.6262 \\
\hline & {$[0.8215]$} & {$[2.0670]$} & {$[1.4880]$} & [0.7998] & [1.1183] & [0.5987] \\
\hline \multirow{2}{*}{$\lambda_{\mathcal{G}}^{C}$} & -0.1804 & -2.9451 & 7.0781 & -1.3884 & 1.1313 & 0.7776 \\
\hline & {$[2.7210]$} & [3.4981] & [3.6293] & {$[1.5051]$} & {$[2.7034]$} & [1.3104] \\
\hline \multirow{2}{*}{$\beta \lambda_{\mathcal{G}}^{D}+\gamma \lambda_{\mathcal{G}}^{C}$} & 0.2238 & 0.1938 & -0.1450 & 0.0873 & -0.0014 & 0.0704 \\
\hline & {$[0.1251]$} & [0.1971] & [0.1019] & {$[0.0498]$} & {$[0.0563]$} & {$[0.0530]$} \\
\hline
\end{tabular}

developments, such as further integration of geographic markets via market coupling, will even further promote the importance of bottom-up modeling frameworks (albeit at the cost of increasing complexity). At the same time, increasing transparency as well as more reliable outturn and forecast data released by system operators help market participants face these challenges and allow for more informed trading decisions.

In this paper, we implement a model for electricity pricing that takes these developments into account by integrating forward-looking information on expected levels of electricity demand and available system capacity. Although hard to compare with other pricing studies that focus on different markets or periods, the derivatives pricing performance of our proposed approach is shown to be very robust and reliable. Importantly, we find that out-of-sample pricing errors can be reduced significantly by making use of forward-looking information. Especially during times of very tight reserve margins, as witnessed for the British market in 2008, capacity forecasts are of crucial importance in order to track sudden outageinduced changes in forward pricing levels and, therefore, significantly reduce pricing errors. However, we have also found that if spare capacities or, equivalently, tightness of the system is not perceived as playing a "fundamental" role, the advantage of employing capacity forecasts reduces and, in some instances, may even lead to marginally lower pricing performance. This is also strongly supported by our findings that capacity forecasts are generally less accurate on average than demand forecasts. Nevertheless, it is still beneficial to keep using demand forecasts rather than using no forecasts at all. This is especially true for the pricing of forwards during the years 2009-2011, where the dynamics of natural gas prices are the main fundamental driver so that demand and capacity only play a subordinate role for pricing.

Given the above mentioned challenges and future developments, there is ample room for further research in the field of structural electricity price modeling. First, it would be interesting to conduct empirical pricing studies for other electricity markets as well. Given that structural electricity price models may always appear somewhat "tailored" to capture the characteristics of a specific electricity market, it would be interesting to see how these types of models perform empirically in those markets where merit-order dynamics are different. Second, given that our model is cast in a log-normal setting, it is equally well-suited to option pricing like other previously proposed fundamental models (see, e.g., Carmona et al., 2013). Further empirical studies might not only investigate the impact of using forward-looking information on option pricing performance, but also focus on the question of how pricing performance is affected depending on whether a 1- or 2-fuel model is used. Finally, the continued shift towards renewable energy sources in the generation mix of many European power markets poses new and highly complex challenges regarding the forecasting of availability levels of intermittent generation, such as for wind or solar power. These forecasts will play an indispensable role especially when modeling geographic markets that are highly interconnected with each other, so that abundant supplies are likely to "spill over" across borders and impact price levels in neighboring markets.

\section{Appendix A}

\section{A.1. Conditional expectations based on enlarged filtrations under the historical measure}

Let $\left(\Omega, \mathcal{F}^{D}, \mathbb{F}^{D}=\left(\mathcal{F}^{D}\right)_{t \in\left[0, T^{\star}\right]}, \mathbb{P}\right)$ be a filtered probability space and $q_{t}$ be specified as in Eq. (2). Assume that $\mathbb{E}^{\mathbb{P}}\left[q_{T_{1}} \mid \mathcal{G}_{t}^{D}\right]$ and $\mathbb{E}^{\mathbb{P}}\left[q_{T_{2}} \mid \mathcal{G}_{t}^{D}\right]$ (with $\mathcal{F}_{t}^{D} \subset \mathcal{G}_{t}^{D}$ ) are available from the system operator. Before computing a forecast of expected electricity demand at time $T$ with $t \leq T_{1} \leq T \leq T_{2}$, we first derive relevant formulae under the assumption that only one forecast point 
for $T_{1}$ is given by the system operator, hence neglecting for the time being the existence of $\mathbb{E}^{\mathbb{P}}\left[q_{T_{2}} \mid \mathcal{G}_{t}^{D}\right]$, and that a forecast of electricity demand is needed for time $T$ with $t \leq T \leq T_{1}$. Formally, this can be expressed as follows:

$$
\mathbb{E}^{\mathbb{P}}\left[q_{T} \mid \mathcal{G}_{t}^{D}\right]=q_{t} \mathrm{e}^{-\kappa^{D}(T-t)}+\sigma^{D} \mathbb{E}^{\mathbb{P}}\left[\int_{t}^{T} \mathrm{e}^{\varphi(s)} \mathrm{e}^{-\kappa^{D}(T-s)} \mathrm{d} B_{s}^{D} \mid \mathcal{G}_{t}^{D}\right] .
$$

In order to manipulate the conditional expectation on the RHS of (21), a standard approach (see, e.g., Protter, 2004; Biagini and Oksendal, 2005) is to exploit the semi-martingale property of $B_{t}^{D}$ with respect to $\mathcal{G}_{t}$, i.e., to decompose $B_{t}^{D}$ as follows:

$$
B_{t}^{D}=\hat{B}_{t}^{D}+A(t)
$$

where $\hat{B}_{t}^{D}$ is a $\mathcal{G}_{t}^{D}$-martingale (standard Brownian motion) and $A(t)$ a continuous $\mathcal{G}_{t}^{D}$-adapted process of finite variation, commonly referred to as the "information drift". Following Hu (2011) and Di Nunno et al. (2006), $\hat{B}_{t}^{D}$ in Eq. (22) can be written more explicitly as

$$
\hat{B}_{t}^{D}=B_{t}^{D}-\underbrace{\int_{0}^{t} b_{t}(s) B_{s}^{D} \mathrm{~d} s}_{A_{1}(t)}-\underbrace{\int_{0}^{t} a(s)\left(\mathbb{E}^{\mathbb{P}}\left[Y \mid \mathcal{G}_{s}^{D}\right]-\rho^{\prime}(s) B_{s}^{D}\right) \mathrm{d} s}_{A_{2}(t)},
$$

with $A(t)=A_{1}(t)+A_{2}(t)$. Following Theorem A.1 in Benth and Meyer-Brandis (2009) or, equivalently, Theorem 3.1 in Hu (2011), $a(s)$ and $b_{t}(s)$ in above Eq. (23) are given as follows:

$$
\begin{aligned}
& a(s)=\frac{\rho^{\prime}(s)}{\tau-\int_{0}^{s}\left(\rho^{\prime}(u)\right)^{2} \mathrm{~d} u}, \\
& b_{t}(s)=\rho^{\prime \prime}(s) \int_{s}^{t} \frac{\rho^{\prime}(v)}{\tau-\int_{0}^{v}\left(\rho^{\prime}(u)\right)^{2} \mathrm{~d} u} \mathrm{~d} v,
\end{aligned}
$$

where $\rho(t)=\mathbb{E}^{\mathbb{P}}\left[B_{t}^{D} Y\right]$ is twice continuously differentiable, $\tau=\mathbb{E}^{\mathbb{P}}\left[Y^{2}\right]$ and $Y$ is a centered Gaussian random variable with $Y=\int_{0}^{T_{1}} \mathrm{e}^{\varphi(s)} \mathrm{e}^{\kappa^{D_{s}}} \mathrm{~d} B_{s}^{D}=\int_{0}^{T_{1}} \mathrm{e}^{\theta \sin (2 \pi(k s+\zeta))} \mathrm{e}^{\mathrm{K}^{D} s} \mathrm{~d} B_{s}^{D}$.

Focusing on $A_{1}(t)$ and since $b_{s}(s)=0$, it holds that

$$
\begin{aligned}
\int_{0}^{t} b_{t}(s) B_{s}^{D} \mathrm{~d} s & =\int_{0}^{t} \int_{0}^{s} \frac{\partial b_{s}}{\partial s}(u) B_{u}^{D} \mathrm{~d} u \mathrm{~d} s \\
& =\int_{0}^{t} a(s)\left[\int_{0}^{s} \rho^{\prime \prime}(u) B_{u}^{D} \mathrm{~d} u\right] \mathrm{d} s \\
\int_{0}^{t} b_{t}(s) B_{s}^{D} \mathrm{~d} s & =\int_{0}^{t} a(s)\left[\rho^{\prime}(s) B_{s}^{D}-\int_{0}^{s} \rho^{\prime}(u) \mathrm{d} B_{u}^{D}\right] \mathrm{d} s,
\end{aligned}
$$

where Eq. (27) is derived from Eq. (26) by applying Itô's lemma to $\rho^{\prime}(s) B_{s}^{D}$. Based on the above, Eq. (23) can now be rearranged to yield

$$
\hat{B}_{t}^{D}=B_{t}^{D}-\underbrace{\int_{0}^{t} a(s)\left(\mathbb{E}^{\mathbb{P}}\left[Y \mid \mathcal{G}_{s}^{D}\right]-\int_{0}^{s} \rho^{\prime}(u) \mathrm{d} B_{u}^{D}\right) \mathrm{d} s}_{A(t)} .
$$

Given above definition of $Y$, and since it can be shown that $\rho^{\prime}(t)=\mathrm{e}^{\varphi(t)} \mathrm{e}^{\kappa^{D} t}$, the information drift $A(t)$ can be further simplified, so that Eq. (28) now reads

$$
\begin{aligned}
\hat{B}_{t}^{D} & =B_{t}^{D}-\int_{0}^{t} a(s) \mathbb{E}^{\mathbb{P}}\left[\int_{s}^{T_{1}} \mathrm{e}^{\varphi(u)} \mathrm{e}^{\kappa^{D} u} \mathrm{~d} B_{u}^{D} \mid \mathcal{G}_{s}^{D}\right] \mathrm{d} s \\
& =B_{t}^{D}-\int_{0}^{t} a(s) \mathbb{E}^{\mathbb{P}}\left[\int_{s}^{T_{1}} \rho^{\prime}(u) \mathrm{d} B_{u}^{D} \mid \mathcal{G}_{s}^{D}\right] \mathrm{d} s \\
\hat{B}_{t}^{D} & =B_{t}^{D}-\underbrace{\mathbb{E}^{\mathbb{P}}\left[\int_{t}^{T_{1}} \rho^{\prime}(u) \mathrm{d} B_{u}^{D} \mid \mathcal{G}_{t}^{D}\right] \int_{0}^{t} a(s) \exp \left(-\int_{t}^{s} \rho^{\prime}(v) a(v) \mathrm{d} v\right) \mathrm{d} s}_{A(t)},
\end{aligned}
$$

where Eq. (30) is derived from Eq. (29) based on Proposition A.3 in Benth and Meyer-Brandis (2009). Hence, in our initial setting of Eq. (21) where a demand forecast $\mathbb{E}^{\mathbb{P}}\left[q_{T} \mid \mathcal{G}_{t}^{D}\right]$ is to be determined that is consistent with the exogenously given forecast point relating to $T_{1}$, this can now be computed as follows:

$$
\mathbb{E}^{\mathbb{P}}\left[q_{T} \mid \mathcal{G}_{t}^{D}\right]=q_{t} \mathrm{e}^{-\kappa^{D}(T-t)}+\underbrace{\sigma^{D} \mathbb{E}^{\mathbb{P}}\left[\int_{t}^{T} \mathrm{e}^{\varphi(s)} \mathrm{e}^{-\kappa^{D}(T-s)} \mathrm{d} B_{s}^{D} \mid \mathcal{G}_{t}^{D}\right]}_{I_{g}(t, T)}
$$




$$
\begin{aligned}
& \mathbb{E}^{\mathbb{P}}\left[q_{T} \mid \mathcal{G}_{t}^{D}\right]=q_{t} \mathrm{e}^{-\kappa^{D}(T-t)}+\sigma^{D} \mathrm{e}^{-\kappa^{D} T} \mathbb{E}^{\mathbb{P}}\left[\int_{t}^{T} \rho^{\prime}(s) \mathrm{d} B_{s}^{D} \mid \mathcal{G}_{t}^{D}\right] \\
& =q_{t} \mathrm{e}^{-\kappa^{D}(T-t)}+\sigma^{D} \mathrm{e}^{-\kappa^{D} T} \mathbb{E}^{\mathbb{P}}\left[\int_{t}^{T} \rho^{\prime}(s) \mathrm{d}\left(\hat{B}_{s}^{D}+A(s)\right) \mid \mathcal{G}_{t}^{D}\right] \\
& =q_{t} \mathrm{e}^{-\kappa^{D}(T-t)}+\sigma^{D} \mathrm{e}^{-\kappa^{D} T} \int_{t}^{T} \rho^{\prime}(s) \mathrm{d} A(s) \\
& =q_{t} \mathrm{e}^{-\kappa^{D}(T-t)}+\sigma^{D} \mathrm{e}^{-\kappa^{D} T} \underbrace{\mathbb{E}^{\mathbb{P}}\left[\int_{t}^{T_{1}} \rho^{\prime}(u) \mathrm{d} B_{u}^{D} \mid \mathcal{G}_{t}^{D}\right]}_{(\star)} \int_{t}^{\int_{t}^{T}} \underbrace{\rho^{\prime}(s) a(s) \exp \left(-\int_{t}^{s} \rho^{\prime}(v) a(v) \mathrm{d} v\right)}_{f(s)} \mathrm{d} s .
\end{aligned}
$$

Note that the term $I_{\mathcal{G}}(t, T)$ is also referred to as information premium which is defined as $\mathbb{E}^{\mathbb{P}}\left[q_{T} \mid \mathcal{G}_{t}^{D}\right]-\mathbb{E}^{\mathbb{P}}\left[q_{T} \mid \mathcal{F}_{t}^{D}\right]$. The term $(\star)$, in turn, can be extracted from the given forecast as follows:

$$
(\star)=\frac{1}{\sigma^{D}}\left(\mathrm{e}^{\kappa^{D} T_{1}} \mathbb{E}^{\mathbb{P}}\left[q_{T_{1}} \mid \mathcal{G}_{t}^{D}\right]-q_{t} \mathrm{e}^{\kappa^{D} t}\right) .
$$

The integral in the second term on the RHS of Eq. (33) can be further simplified if volatility is constant, as is the case for the dynamics of the capacity process in Eq. (6). In the case of the seasonal volatility function for the demand process as specified in Eq. (4), however, no analytic solutions for the integral exist; still, it can be approximated computationally in an efficient way by using standard numerical integration techniques.

Having outlined the general procedure for the case $T \leq T_{1}$, we now turn to the more relevant case where $\mathbb{E}^{\mathbb{P}}\left[q_{T_{1}} \mid \mathcal{G}_{t}^{D}\right]$ and $\mathbb{E}^{\mathbb{P}}\left[q_{T_{2}} \mid \mathcal{G}_{t}^{D}\right]$ (with $\mathcal{F}_{t}^{D} \subset \mathcal{G}_{t}^{D}$ ) are released by the system operator and a forecast $\mathbb{E}^{\mathbb{P}}\left[q_{T} \mid \mathcal{G}_{t}^{D}\right]$ needs to be computed with $t \leq T_{1} \leq T \leq T_{2}$. We proceed as follows:

$$
\mathbb{E}^{\mathbb{P}}\left[q_{T} \mid \mathcal{G}_{t}^{D}\right]=\mathbb{E}^{\mathbb{P}}[q_{T_{1}}+\underbrace{\mathbb{E}^{\mathbb{P}}\left[q_{T}-q_{T_{1}} \mid \mathcal{G}_{T_{1}}^{D}\right]}_{(\star \star)} \mid \mathcal{G}_{t}^{D}] .
$$

Re-arranging $(\star \star)$ and taking out what is known, i.e. $\mathcal{G}_{T_{1}}^{D}$-measurable, we get

$$
\mathbb{E}^{\mathbb{P}}\left[q_{T}-q_{T_{1}} \mid \mathcal{G}_{T_{1}}^{D}\right]=q_{T_{1}}\left(\mathrm{e}^{-\kappa^{D}\left(T-T_{1}\right)}-1\right)+\sigma^{D} \mathbb{E}^{\mathbb{P}}\left[\int_{T_{1}}^{T} \mathrm{e}^{\varphi(s)} \mathrm{e}^{-\kappa^{D}(T-s)} \mathrm{d} B_{s}^{D} \mid \mathcal{G}_{T_{1}}^{D}\right] .
$$

Combining Eqs. (35) and (36) and using iterated conditioning now yields

$$
\begin{aligned}
\mathbb{E}^{\mathbb{P}}\left[q_{T} \mid \mathcal{G}_{t}^{D}\right] & =\mathbb{E}^{\mathbb{P}}\left[q_{T_{1}} \mid \mathcal{G}_{t}^{D}\right] \mathrm{e}^{-\kappa^{D}\left(T-T_{1}\right)}+\mathbb{E}^{\mathbb{P}}\left\{\sigma^{D} \mathbb{E}^{\mathbb{P}}\left[\int_{T_{1}}^{T} \mathrm{e}^{\varphi(s)} \mathrm{e}^{-\kappa^{D}(T-s)} \mathrm{d} B_{s}^{D} \mid \mathcal{G}_{T_{1}}^{D}\right] \mid \mathcal{G}_{t}^{D}\right\} \\
& =\mathbb{E}^{\mathbb{P}}\left[q_{T_{1}} \mid \mathcal{G}_{t}^{D}\right] \mathrm{e}^{-\kappa^{D}\left(T-T_{1}\right)}+\mathbb{E}^{\mathbb{P}}\left[I_{\mathcal{G}}\left(T_{1}, T\right) \mid \mathcal{G}_{t}^{D}\right] .
\end{aligned}
$$

The term $\mathbb{E}^{\mathbb{P}}\left[I_{\mathcal{G}}\left(T_{1}, T\right) \mid \mathcal{G}_{t}^{D}\right]$ in Eq. (37), however, can be manipulated similarly to Eqs. (31)-(33):

$$
\begin{aligned}
\mathbb{E}^{\mathbb{P}}\left[I_{\mathcal{G}}\left(T_{1}, T\right) \mid \mathcal{G}_{t}\right] & =\mathbb{E}^{\mathbb{P}}\left\{\sigma^{D} \mathrm{e}^{-\kappa^{D} T} \mathbb{E}^{\mathbb{P}}\left[\int_{T_{1}}^{T_{2}} \rho^{\prime}(u) \mathrm{d} B_{u}^{D} \mid \mathcal{G}_{T_{1}}^{D}\right] \int_{T_{1}}^{T} f(s) \mathrm{d} s \mid \mathcal{G}_{t}^{D}\right\} \\
& =\sigma^{D} \mathrm{e}^{-\kappa^{D} T} \underbrace{\mathbb{E}^{\mathbb{P}}\left[\int_{T_{1}}^{T_{2}} \rho^{\prime}(u) \mathrm{d} B_{u}^{D} \mid \mathcal{G}_{t}^{D}\right]}_{(\star \star \star)} \int_{T_{1}}^{T} f(s) \mathrm{d} s .
\end{aligned}
$$

Analogous to Eq. (34), the term $(\star \star \star)$ can be backed out from the given forecast points relating to $T_{1}$ and $T_{2}$

$$
(\star \star \star)=\frac{1}{\sigma^{D}}\left(\mathrm{e}^{\kappa^{D} T_{2}} \mathbb{E}^{\mathbb{P}}\left[q_{T_{2}} \mid \mathcal{G}_{t}^{D}\right]-\mathrm{e}^{\kappa^{D} T_{1}} \mathbb{E}^{\mathbb{P}}\left[q_{T_{1}} \mid \mathcal{G}_{t}^{D}\right]\right) .
$$

\section{A.2. Conditional expectations based on enlarged filtrations under an equivalent risk-neutral measure}

For derivatives pricing purposes, and based on Eq. (14), conditional expectations $\mathbb{E}^{\mathbb{Q}}\left[\cdot \mid \mathcal{G}_{t}\right]$ and variances $\mathbb{V}^{\mathbb{Q}}\left[\cdot \mid \mathcal{G}_{t}\right]$ under the enlarged filtration $\left(\mathcal{G}_{t}\right)_{t \in\left[0, T^{\star}\right]}$ and a risk-neutral measure $\mathbb{Q}$ need to be computed for both demand and capacity processes $D_{t}$ and $C_{t}$, respectively.

Defining $A(t)=\int_{0}^{t} \vartheta(s) \mathrm{d} s$, and based on the manipulations in the previous subsection, the $\mathcal{G}^{D}$-adapted dynamics for $D_{t}$ can be stated as below (see Eq. (2)):

$$
\mathrm{d} q_{t}=-\kappa^{D}\left(q_{t}-\frac{\sigma^{D} \mathrm{e}^{\varphi(t)}}{\kappa^{D}} \vartheta(t)\right) \mathrm{d} t+\sigma^{D} \mathrm{e}^{\varphi(t)} \mathrm{d} \hat{B}_{t}^{D}
$$


where $\hat{B}_{t}^{D}$ is a $\mathcal{G}_{t}^{D}$-adapted standard P-Brownian motion. ${ }^{39}$ Applying Girsanov's theorem, and given that our market setting is inherently incomplete, we assume that under a suitably chosen risk-neutral measure $\mathbb{Q}, \hat{B}_{t}^{D}$ is a semi-martingale and decomposes as follows:

$$
\hat{B}_{t}^{D}=\check{B}_{t}^{D}+\Lambda_{\mathcal{G}}^{D}(t)
$$

where $\breve{B}_{t}^{D}$ is a $\mathcal{G}_{t}^{D}$-adapted standard $\mathbb{Q}$-Brownian motion and $\Lambda_{\mathcal{G}}^{D}(t)=\int_{0}^{t} \lambda_{\mathcal{G}}^{D}(s)$ ds is a finite variation process governing the change of measure as market price of demand risk. The risk-neutral dynamics for $D_{t}$ under the enlarged filtration now are

$$
\mathrm{d} q_{t}=-\kappa^{D}\left(q_{t}-\frac{\sigma^{D} \mathrm{e}^{\varphi(t)}}{\kappa^{D}}\left(\vartheta(t)+\lambda_{\mathcal{G}}^{D}(t)\right)\right) \mathrm{d} t+\sigma^{D} \mathrm{e}^{\varphi(t)} \mathrm{d} \check{B}_{t}^{D}
$$

where conditional expectation $\mathbb{E}^{\mathbb{Q}}\left[\cdot \mid \mathcal{G}_{t}\right]$ and variance $\mathbb{V}^{\mathbb{Q}}\left[\cdot \mid \mathcal{G}_{t}\right]$ are then derived in the standard way. As outlined in Section 4 , the market price of risk will be assumed constant and inferred from price quotes of traded derivative contracts. Depending on whether or not forward-looking information will be used, it will be referred to as $\lambda_{\mathcal{G}}^{D}$ or $\lambda_{\mathcal{F}}^{D}$, respectively.

\section{References}

Aïd, R., Campi, L., Huu, A.N., Touzi, N., 2009. A structural risk-neutral model of electricity prices. Int. J. Theoret. Appl. Finance 12 (7), $925-947$. Aïd, R., Chemla, G., Porchet, A., Touzi, N., 2011. Hedging and vertical integration in electricity markets. Manag. Sci. 57 (8), $1438-1452$.

Aïd, R., Campi, L., Langrené, N., 2013. A structural risk-neutral model for pricing and hedging power derivatives. Math. Finance 23 (3), $387-438$. Back, J., Prokopczuk, M., Rudolf, M., 2013. Seasonality and the valuation of commodity options. J. Bank. Finance 37 (2), $273-290$.

Benth, F.E., Koekebakker, S., 2008. Stochastic modeling of financial electricity contracts. Energy Econ. 30 (3), 1116-1157.

Benth, F.E., Meyer-Brandis, T., 2009. The information premium for non-storable commodities. J. Energy Mark. 2 (3), 111-140.

Benth, F.E., Biegler-König, R., Kiesel, R., 2013. An empirical study of the information premium on electricity markets. Energy Econ. 36, 55-77. Bessembinder, H., Lemmon, M.L., 2002. Equilibrium pricing and optimal hedging in electricity forward markets. J. Finance 57 (3), $1347-1382$. Biagini, F., Oksendal, B., 2005. A general stochastic calculus approach to insider trading. Appl. Math. Optim. 52 (2), $167-181$.

Bierbrauer, M., Menn, C., Rachev, S., Trueck, S., 2007. Spot and derivative pricing in the EEX power market. J. Bank. Finance 31 (11), $3462-3485$. Blumsack, S., 2014. Dash for gas, 21st-century style!. Elements 10, 265-270.

Bordignon, S., Bunn, D.W., Lisi, F., Nan, F., 2013. Combining day-ahead forecasts for British electricity prices. Energy Econ. 35 (1), 88-103.

Buehler, W., Mueller-Mehrbach, J., 2007. Dynamic Equilibrium Valuation of Electricity Futures. Working Paper, Universität Mannheim, 2007.

Buehler, W., Mueller-Mehrbach, J., 2009. Valuation of Electricity Futures: Reduced-Form Vs. Dynamic Equilibrium Models. Working Paper, Universität Mannheim.

Bunn, D.W. Fezzi, C.,2008. A vector error correction model of the interactions among gas, electricity and carbon prices: an application to the cases of Germany and the United Kingdom. In: Gulli, F. (Ed.), Markets for Carbon and Power Pricing in Europe: Theoretical Issues and Empirical Analyses, Edward Elgar, Cheltenham, UK.

Burger, M., Klar, B., Mueller, A., Schindlmayr, G., 2004. A spot market model for pricing derivatives in electricity markets. Quant. Finance 4 (1), 109-122. Carmona, R., Coulon, M., 2012. A survey of commodity markets and structural model for electricity prices. In: Benth, F.E. (Ed.), Financial Engineering for Energy Asset Management and Hedging in Commodity Markets; Proceedings from the Special Thematic Year at the Wolfgang Pauli Institute, Vienna.

Carmona, R., Coulon, M., Schwarz, D., 2013. Electricity price modeling and asset valuation: a multi-fuel structural approach. Math. Finan. Econ. 7 (2), $167-202$.

Cartea, A., Villaplana, P., 2008. Spot price modeling and the valuation of electricity forward contracts: the role of demand and capacity. J. Bank. Finance 32 (12), 2502-2519.

Cartea, A., Williams, T., 2008. UK gas markets: the market price of risk and applications to multiple interruptible supply contracts. Energy Econ. 30 (3), $829-846$.

Cartea, A., Figueroa, M.G., Geman, H., 2009. Modelling electricity prices with forward looking capacity constraints. Appl. Math. Finance 16 (2), $103-122$. Clewlow, L., Strickland, C., 2000. Energy Derivatives: Pricing and Risk Management. Lacima Productions, London.

Coulon, M., Howison, S., 2009. Stochastic behaviour of the electricity bid stack: from fundamental drivers to power prices. J. Energy Mark. 2 (1), 29-69.

Coulon, M., Powell, W.B., Sircar, R., 2013. A model for hedging load and price risk in the Texas electricity market. Energy Econ. 40, 976-988.

Cox, J.C., Ross, S.A., 1976. The valuation of options for alternative stochastic processes. J. Finan. Econ. 3 (1-2), 145-166.

Di Nunno, G., Meyer-Brandis, T., Oksendal, B., Proske, F., 2006. Optimal portfolio for an insider in a market driven by Lévy processes. Quant. Finance 6 (1), $83-94$.

Dorfleitner, G., Wimmer, M., 2010. The pricing of temperature futures at the Chicago Mercantile Exchange. J. Bank. Finance 34 (6), $1360-1370$.

Eydeland, A., Wolyniec, K., 2002. Energy and Power Risk Management: New Developments in Modeling, Pricing, and Hedging. John Wiley and Sons, New York.

Fanone, E., Gamba, A., Prokopczuk, M., 2013. The case of negative day-ahead electricity prices. Energy Econ. 35 (1), $22-34$.

Geman, H., Nguyen, V.-N., 2005. Soybean inventory and forward curve dynamics. Manag. Sci. 51 (7), $1076-1091$.

Haerdle, W., Lopez-Cabrera, B., Ritter, M., 2012. Forecast Based Pricing of Weather Derivatives. Discussion Paper, SFB 649, Humboldt-Universität zu Berlin. Harrison, J.M., Kreps, D.M., 1979. Martingales and arbitrage in multiperiod securities markets. J. Econ. Theory 20 (3), $381-408$.

Hu, Y., 2011. An enlargement of filtration for Brownian motion. Acta Math. Sci. 31B (5), 1671-1678.

Janczura, J., Weron, R., 2010. An empirical comparison of alternate regime-switching models for electricity spot prices. Energy Econ. 32 (5), 1059-1073.

Jewson, S., Caballero, R., 2003. The use of weather forecasts in the pricing of weather derivatives. Meteorol. Appl. 10 (4), $377-389$.

Johnson, B., Barz, G., 1999. Selecting stochastic processes for modelling electricity prices. In: Energy Modelling and the Management of Uncertainty, Risk Publication, London, pp. 3-21.

Kanamura, T., Ohashi, K., 2007. A structural model for electricity prices with spikes: measurement of spike risk and optimal policies for hydropower plant operation. Energy Econ. 29 (5), 1010-1032.

Karakatsani, N.V., Bunn, D.W., 2008. Forecasting electricity prices: the impact of fundamentals and time-varying coefficients. Int. J. Forecast. 24 (4), 764-785. Koekebakker, S., Ollmar, F., 2005. Forward curve dynamics in the Nordic electricity market. Manag. Finance 31 (6), $72-93$.

Lucia, J.J., Schwartz, E.S., 2002. Electricity prices and power derivatives: evidence from the Nordic power exchange. Rev. Deriv. Res. 5 (1), 5-50.

Lyle, M.R., Elliott, R.J., 2009. A 'simple' hybrid model for power derivatives. Energy Econ. 31 (5), $757-767$.

Lynch, K.J., Brayshaw, D.J., Charlton-Perez, A., 2014. Verification of European subseasonal wind speed forecasts. Mon. Weather Rev. 142 (8), $2978-2990$.

Misiorek, A., Trueck, S., Weron, R., 2006. Point and interval forecasting of spot electricity prices: linear vs. non-linear time series models. Stud. Nonlinear Dyn. Econom. 10 (3). Article 2.

\footnotetext{
${ }^{39}$ Recall that we assume the filtration $\left(\mathcal{G}_{t}^{C}\right)_{t \in\left[0, T^{\star}\right]}$ to be of such nature that $B_{t}^{D}=\hat{B}_{t}^{D}+A(t)$ is a semi-martingale.
} 
OFGEM. GB Wholesale Electricity Market Liquidity: Summer 2011 Assessment. URL 〈http://www.ofgem.gov.uk〉.

Pirrong, C., 2012. Commodity Price Dynamics: a Structural Approach. Cambridge University Press, Cambridge.

Pirrong, C., Jermakyan, M., 2008. The price of power: the valuation of power and weather derivatives. J. Bank. Finance 32 (12), $2520-2529$.

Protter, P., 2004. Stochastic Integration and Differential Equations. Springer, New York.

Ritter, M., Mußhoff, O., Odening, M., 2011. Meteorological forecasts and the pricing of temperature futures. J. Deriv. 19 (2), 45-60.

Schwartz, E.S., 1997. The stochastic behavior of commodity prices: implications for valuation and hedging. J. Finance 52 (3), $923-973$.

Seifert, J., Uhrig-Homburg, M., 2007. Modelling jumps in electricity prices: theory and empirical evidence. Rev. Deriv. Res. 10 (1), $59-85$.

Skantze, P., Gubina, A., Ilic, M., 2000. Bid-based Stochastic Model for Electricity Prices: the Impact of Fundamental Drivers on Market Dynamics. MIT Energy Laboratory Publication, Cambridge, Massachusetts.

Weron, R., 2006. Modeling and Forecasting Electricity Loads and Prices: a Statistical Approach. Wiley, Chichester.

White, H., 1982. Maximum likelihood estimation of misspecified models. Econometrica 50 (1), 1-25.

Wilson, I.A.G., McGregor, P.G., Infield, D.G., Hall, P.J., 2011. Grid-connected renewables, storage and the UK electricity market. Renew. Energy 36 (8), $2166-2170$. 